\title{
The middle-atmosphere Hadley circulation and equatorial inertial adjustment
}

Article

Published Version

Semeniuk, K. and Shepherd, T. G. (2001) The middleatmosphere Hadley circulation and equatorial inertial adjustment. Journal of the Atmospheric Sciences, 58 (21). pp. 3077-3096. ISSN 1520-0469 doi: https://doi.org/10.1175/15200469(2001)058<3077:TMAHCA>2.0.CO;2 Available at https://centaur.reading.ac.uk/32846/

It is advisable to refer to the publisher's version if you intend to cite from the work. See Guidance on citing.

Published version at: http://dx.doi.org/10.1175/1520-0469(2001)058<3077:TMAHCA>2.0.CO;2

To link to this article DOI: http://dx.doi.org/10.1175/1520-

0469(2001)058<3077:TMAHCA>2.0.CO;2

Publisher: American Meteorological Society

All outputs in CentAUR are protected by Intellectual Property Rights law, including copyright law. Copyright and IPR is retained by the creators or other copyright holders. Terms and conditions for use of this material are defined in the End User Agreement.

www.reading.ac.uk/centaur 
Central Archive at the University of Reading

Reading's research outputs online 


\title{
The Middle-Atmosphere Hadley Circulation and Equatorial Inertial Adjustment
}

\author{
Kirill Semeniuk And Theodore G. Shepherd \\ Department of Physics, University of Toronto, Toronto, Ontario, Canada
}

(Manuscript received 12 May 2000, in final form 19 April 2001)

\begin{abstract}
In the tropical middle atmosphere the climatological radiative equilibrium temperature is inconsistent with gradient-wind balance and the available angular momentum, especially during solstice seasons. Adjustment toward a balanced state results in a type of Hadley circulation that lies outside the "downward control" view of zonally averaged dynamics. This middle-atmosphere Hadley circulation is reexamined here using a zonally symmetric balance model driven through an annual cycle. It is found that the inclusion of a realistic radiation scheme leads to a concentration of the circulation near the stratopause and to its closing off in the mesosphere, with no need for relaxational damping or a rigid lid. The evolving zonal flow is inertially unstable, leading to a rapid process of inertial adjustment, which becomes significant in the mesosphere. This short-circuits the slower process of angular momentum homogenization by the Hadley circulation itself, thereby weakening the latter.

The effect of the meridional circulation associated with extratropical wave drag on the Hadley circulation is considered. It is shown that the two circulations are independent for linear (quasigeostrophic) zonal-mean dynamics, and interact primarily through the advection of temperature and angular momentum. There appears to be no significant coupling in the deep Tropics via temperature advection since the wave-driven circulation is unable to alter meridional temperature gradients in this region. However, the wave-driven circulation can affect the Hadley circulation by advecting angular momentum out of the Tropics.

The validity of the zonally symmetric balance model with parameterized inertial adjustment is tested by comparison with a three-dimensional primitive equations model. Fields from a middle-atmosphere GCM are also examined for evidence of these processes. While many aspects of the GCM circulation are indicative of the middle-atmosphere Hadley circulation, particularly in the upper stratosphere, it appears that the circulation is obscured in the mesosphere and lower stratosphere by other processes.
\end{abstract}

\section{Introduction}

The diabatic circulation plays an important role in the climate of the middle atmosphere as the primary agent of transport of chemically and radiatively active constituents (Andrews et al. 1987). Stratospheric transport is characterized by upwelling in the Tropics with poleward and downward motion in the extratropics. Current understanding of the dynamics of the diabatic circulation holds that it is driven by extratropical wave drag (Holton et al. 1995). However, Dunkerton (1989) has shown that the tropical middle atmosphere can support a Hadley circulation, which does not depend on wave driving or viscosity. The theory of the nonlinear Hadley circulation was originally developed by Held and Hou (1980) for the troposphere and is widely accepted in that context, but its middle-atmosphere counterpart has received relatively little attention. In this paper we revisit the work of Dunkerton and consider the dynamics

Corresponding author address: Dr. Theodore G. Shepherd, Dept. of Physics, University of Toronto, 60 St. George Street, Toronto, ON M5S 1A7, Canada.

E-mail: tgs@atmosp.physics.utoronto.ca of the middle-atmosphere Hadley circulation using less restrictive models.

In the extratropics, angular momentum deposition by waves (loosely termed "wave drag") induces a meridional circulation and temperature change in thermalwind balance with the zonal-wind acceleration (Eliassen 1951). Radiative relaxation counteracts the adiabatic heating/cooling and allows a diabatic circulation to develop. During solstice seasons a steady state is approached where streamlines concentrate beneath wave drag regions and are aligned with the horizontally stratified absolute angular momentum isopleths (Haynes et al. 1991). This is because in steady state, streamlines are prohibited from crossing angular momentum isopleths in regions without mechanical forcing. Consequently, angular momentum conserving processes such as persistent diabatic heating (e.g., a change in the radiative equilibrium temperature) cannot drive a steadystate meridional circulation. Instead, there is only a local temperature change.

This "downward control" of wave drag on the streamlines in steady state depends on the coupling of the zonal wind and temperature through thermal-wind balance. As a result of the weak Coriolis parameter, 
near-equatorial zonal wind forcing has a weak thermal signature and the time it takes to approach steady state diverges at the equator. The quasi-biennial oscillation (QBO) and westerly phase of the semiannual oscillation (SAO) are examples of the nature of the equatorial response, which is to balance wave angular momentum deposition primarily by the acceleration of the zonal wind.

Near the equator it is possible for the radiative equilibrium temperature to be incompatible with thermalwind balance, or more specifically gradient-wind balance. This was pointed out by Dunkerton (1989), who noted that the meridional gradients in the radiative equilibrium temperature near the equator that form during solstice seasons, particularly in the upper stratosphere and lower mesosphere, correspond to an equilibrium state that is not attainable by the balance system due to the finite available angular momentum. The solstice case is an extreme one where the zonal wind corresponding to the radiative equilibrium temperature is singular at the equator where the Coriolis parameter vanishes. The result is a persistent diabatic heating that drives a singlecell meridional circulation. In the absence of wave angular momentum damping, this angular momentum conserving cell would, if allowed to continue forever, destroy the horizontal angular momentum stratification in the Tropics. The solstitial middle-atmosphere Hadley circulation is an equatorially asymmetric variant of the tropospheric Hadley circulation considered by Held and Hou (1980). During equinox seasons the middle-atmosphere radiative equilibrium temperature has sufficient latitudinal curvature in the Tropics to support the tropospheric variant, although it is found in this study to be weak.

There is no a priori reason for gradient-wind balance to hold in the Tropics and the zonal wind singularity problem may not arise at all. However, observations indicate that during solstice seasons the zonal-mean temperature has little or no latitudinal gradient at the equator over the altitude range spanning the middle atmosphere. Simulations using primitive equations models (e.g., GCMs), which are not based on the gradientwind balance assumption, also produce temperature distributions subject to this constraint. These models indicate that for zonal-mean fields, gradient-wind balance is a good approximation in the stratosphere. One objective of this work is to test the validity of the gradientwind balance assumption in the Tropics under solstice conditions, as it is relevant to the development of the middle-atmosphere Hadley circulation.

Some evidence for the existence of the middle-atmosphere Hadley circulation also comes from the latitudinal profile of upwelling in the Tropics, as suggested by Dunkerton (1989). The observed upwelling maximum tends to occur on the summer side of the equator (Randel et al. 1998). This behavior is not consistent with the wave drag distribution inferred from observations, which minimizes in the equatorial stratosphere except for the upper layers (e.g., Rosenlof 1995, Fig. 8). In the absence of other processes, the circulation produced by this wave drag should have an upwelling pattern with a strong maximum in the winter hemisphere and a weak maximum in the summer hemisphere, as is evident from the results of Plumb and Eluszkiewicz (1999). They noted that an additional tropical thermal forcing is necessary to explain the upwelling profile (cf. their Figs. $14 \mathrm{~b}$ and $14 \mathrm{c})$.

The solstitial Hadley circulation advects the absolute angular momentum maximum off the equator into the winter hemisphere, thereby triggering inertial adjustment. Inertial adjustment acts on the absolute angular momentum distribution much like the overturning produced by the Hadley circulation, except on a much shorter timescale. Direct representation of this process was omitted in Dunkerton (1989); instead, a "parabolic approximation" to the streamfunction equation was used to facilitate the solution of the system. Dunkerton found that solutions of his zonal-mean models were insensitive to this approximation in the steady-state limit. We use an inertial adjustment parameterization in our zonal-mean model and find that the resulting redistribution of the zonal wind and temperature has a significant effect on the seasonally evolving Hadley circulation.

Dunkerton $(1989,1991)$ also considered the influence of extratropical wave drag on the Hadley circulation. Based on the "equal-area rule" for the deviation of the temperature from radiative equilibrium, it was argued that remote mechanical forcing can intensify and increase the latitudinal extent of the diabatic circulation. However, it is not clear from the diabatic heating how the Hadley circulation and the wave-driven circulation interact, because the diabatic heating responds to both circulations. It is shown here that the two circulations are independent in the special case of linear (quasigeostrophic) zonal-mean dynamics and Newtonian cooling. The extent to which they are separable in the presence of nonlinearity is more difficult to assess. In this study the focus is on the effect of the wave-driven circulation and wave drag on the Hadley circulation. The sensitivity of the wave-driven circulation to the tropical absolute angular momentum distribution and hence to the Hadley circulation is investigated in the companion paper, Semeniuk and Shepherd (2001).

A description of the numerical model used is given in section 2. This zonal-mean model differs from the one used by Dunkerton in that no attempt is made to close off the Hadley cell in the mesosphere (with either a lid at the stratopause or a friction, or sponge, layer in the mesosphere), and inertial adjustment is explicitly parameterized. Section 3 reexamines the middle-atmosphere Hadley circulation in light of the relaxed model assumptions. The effect of the vertical dependence of radiative damping rate and equilibrium temperature is also considered, using a realistic radiation scheme. It is found that the Hadley circulation undergoes a transition 
in the tropical stratopause region from a weak crossequatorial flow regime in the stratosphere to a strong one in the mesosphere. This transition reflects the vertical distribution of the diabatic heating of the Hadley circulation, and is sensitive to the vertical profile of the radiative damping rate. Tests of gradient-wind balance in the Tropics using a three-dimensional primitive equations model are also presented. The extent of the influence of the wave-driven circulation on the Hadley circulation is revisited in section 4. Some evidence of the Hadley circulation in middle-atmosphere GCMs is described in section 5 .

\section{Model}

The results of this paper are for the most part based on numerical solutions of the transformed Eulerian mean form of the governing equations (e.g., Andrews et al. 1987)

$$
\begin{gathered}
\frac{\partial \bar{u}}{\partial t}+\bar{v}^{*}\left(\frac{1}{a} \frac{\partial \bar{u}}{\partial \phi}-f-\bar{u} \frac{\tan \phi}{a}\right)+\bar{w}^{*} \frac{\partial \bar{u}}{\partial z}=\mathcal{F}, \\
\frac{\partial \bar{T}}{\partial t}+\bar{v}^{*} \frac{1}{a} \frac{\partial \bar{T}}{\partial \phi}+\bar{w}^{*} S=Q, \\
\left(f+2 \bar{u} \frac{\tan \phi}{a}\right) \frac{\partial \bar{u}}{\partial z}=-\frac{R}{a H} \frac{\partial \bar{T}}{\partial \phi}, \\
\frac{1}{a \cos \phi} \frac{\partial\left(\bar{v}^{*} \cos \phi\right)}{\partial \phi}+\frac{1}{\rho} \frac{\partial\left(\rho \bar{w}^{*}\right)}{\partial z}=0,
\end{gathered}
$$

where $a$ is the planetary radius, $f=2 \Omega \sin \phi$ is the Coriolis parameter, $\rho=\rho_{0} \exp (-z / H)$ is the reference density profile with $H$ the constant density scale height, $\left(\bar{v}^{*}, \bar{w}^{*}\right)$ is the residual circulation, and $S \equiv \partial \bar{T} / \partial z+$ $(\kappa / H) \bar{T}$ is the static stability parameter. The EliassenPalm flux divergence representing forcing by wave drag is denoted by $\mathcal{F}$, while $Q$ denotes the diabatic heating. An eddy heat flux term has been neglected on the righthand side of (2); this term is conventionally assumed to be small. With this assumption $\left(\bar{v}^{*}, \bar{w}^{*}\right)$ is equivalent to what is known as the transient diabatic circulation. The diabatic heating can be represented by a Newtonian cooling to some time-dependent radiative equilibrium temperature, or by a more comprehensive radiative transfer scheme. Equation (3) represents thermal-wind balance (a combination of gradient-wind and hydrostatic balance), while (4) represents mass continuity. The surface friction scheme is based on the stress condition of Held and Hou (1980) with a drag coefficient of $C=$ $0.005 \mathrm{~s}^{-1}$. Vertical and horizontal diffusion are included in (1) and (2) with constant coefficients of 0.25 and $5000 \mathrm{~m}^{2} \mathrm{~s}^{-1}$, respectively, to control numerical instability. The solutions were not sensitive to this choice for the diffusion coefficients and were very close to those obtained with much smaller values.

Mass continuity allows a streamfunction representa- tion for the residual circulation. Defining $\partial \psi / \partial z=-\rho$ $\cos \phi \bar{v}^{*}$ and $\partial \psi / \partial \phi=a \rho \cos \phi \bar{w}^{*}$ and using thermalwind balance, (1)-(4) can be combined into a single instantaneous equation (see appendix A for details):

$$
\begin{aligned}
\mathcal{L}[\psi] & \equiv A \frac{\partial^{2} \psi}{\partial \phi^{2}}+B \frac{\partial^{2} \psi}{\partial \phi \partial z}+C \frac{\partial^{2} \psi}{\partial z^{2}}+D \frac{\partial \psi}{\partial \phi}+E \frac{\partial \psi}{\partial z} \\
& =F
\end{aligned}
$$

where the coefficients of the $\psi$ terms are functionals of $\bar{u}$ and $\bar{T}$, and $F$ is a functional of $\bar{u}, \mathcal{F}$ and $Q$.

The operator on the left-hand side of $(5), \mathcal{L}$, can be mixed elliptic, parabolic, or hyperbolic depending on the local sign of $B^{2}-4 A C$, which is determined by the distributions of $\bar{u}$ and $\bar{T}$. The behavior of the coefficients is largely determined by the planetary rotation: the latter dominates the contribution of $\bar{u}$ and $\bar{T}$ to $\mathcal{L}$ in the extratropics leading to ellipticity, but weakens in the Tropics allowing parabolic and hyperbolic regions to form.

In the middle atmosphere, hyperbolic regions are characterized by $\partial \bar{u} / \partial \phi-a f-\bar{u} \tan \phi$ positive for $\phi$ $>0$ or negative for $\phi<0$, in which case the coefficient of $\bar{v}^{*}$ in (1) has an opposite sign to $-f$. These regions can form if the absolute angular momentum maximum is moved off the equator. As will be shown in section 3 , the maximum is shifted into the winter hemisphere in the upper stratosphere and above by cross-equatorial advection, resulting in a hyperbolic region on the winter side of the equator.

The formation of hyperbolic regions represents a breakdown of the assumptions underlying the balance model (1)-(4), since such regions are symmetrically unstable (Eliassen 1951); the basic state, although in gradient-wind and hydrostatic balance, cannot be realized. Symmetric instability and the associated adjustment are not captured by the balance model and have to be parameterized. The adjustment prevents $\mathcal{L}$ from becoming hyperbolic, but $\mathcal{L}$ can be parabolic in regions of marginal stability.

The parameterization implemented here is based on the one used by Holton and Wehrbein (1980) and presumes that symmetric instability is primarily inertial instability; the details are given in appendix B. It involves a conservative redistribution of the zonal wind in latitude at every time step in regions where

$$
f\left(f-\frac{1}{a} \frac{\partial \bar{u}}{\partial \phi}\right)<0 .
$$

The $\bar{u} \tan \phi / a$ term is neglected since it was found to contribute to numerical instability. The result of this approximation is a latitudinal $\bar{m}$ profile with a smooth curvature near the poleward edge of the adjustment region, where $\bar{u}$ is large, instead of a sharp edge. At lower latitudes the adjustment scheme yields an $\bar{m}$ profile that is linear but with a small negative (positive) slope in the Northern (Southern) Hemisphere. This redistribution asymmetry, or overadjustment, is not due to the ap- 
proximation above but depends on model resolution. It can be avoided altogether by adjusting $\bar{m}$ rather than $\bar{u}$. However, the model solution with large parabolic regions developed certain features that led to numerical instability. We use the overadjusting scheme based on the consideration that the difference in $\bar{u}$ between the overadjusted case and the parabolic case is small.

The adjustment scheme is designed to redistribute some of the absolute angular momentum into the summer hemisphere to avoid the formation of a discontinuity at the equator. This is motivated by three-dimensional model simulations and observations, which do not show a sharp transition in $\bar{m}$ at the equator during solstice seasons (see later sections). The temperature is also adjusted to maintain thermal-wind balance with the modified zonal wind distribution, although it was found that the model formulation is robust enough not to require this.

In the model of Dunkerton (1989) the solvability of the system is maintained at every time step by modifying the streamfunction operator to be parabolic in hyperbolic regions. The zonal wind and temperature were not adjusted, although a solution with inertial adjustment was presented for comparison. Our model formulation was found to be sensitive to large values of cross-equatorial angular momentum flux that develop in the absence of adjustment, so this method was not used.

The numerical scheme used to solve the system given by Eqs. (1), (2), and (5) is inspired by that of Kinnersley (1996). The system is discretized and then the streamfunction equation is obtained by imposing the condition that thermal-wind balance hold at the end of each time step. The aim is to preserve thermal-wind balance while using both prognostic equations (1) and (2). This avoids the need to obtain $\bar{u}$ from $\bar{T}$ or vice versa, which is uncertain at every timestep up to some additive function of height or latitude.

The particular approach used here to obtain the streamfunction equation is to replace (1) with a prognostic equation for the variable $(\bar{m} / a)^{2}=\cos ^{2} \phi(\bar{u}+a \Omega$ $\cos \phi)^{2}$. This involves no loss of sign information for $\bar{u}$ since $\bar{m}$, the absolute angular momentum, is positive under climatological conditions so $\bar{u}$ can be uniquely determined from it. This formulation does restrict the amplitude of forcing that can be used in the model. The advantage is that the thermal-wind relation between $(\bar{m} / a)^{2}$ and $\bar{T}$ is linear (Stevens 1983). Therefore the streamfunction equation is also linear and the necessity for a nonlinear iterative solution at every time step is avoided. The streamfunction is obtained at every time step using the MUDPACK multigrid solver (numerical library created by John C. Adams at the National Center for Atmospheric Research). This elliptic solver can handle parabolicity in $\mathcal{L}$ over portions of the domain.

\section{The Hadley circulation}

The basics of the solstitial tropical angular-momentum-conserving circulation described by Dunkerton
(1989) are presented in this section. Some aspects that were not addressed previously in this context are considered. These pertain to the effects of a more realistic radiative heating scheme, and to the distinct roles of inertial adjustment and the diabatic circulation in redistributing $\bar{m}$ within the Tropics. In addition, the balance model solutions are compared with those of a threedimensional primitive equations model.

\section{a. Theory}

During solstice seasons there are strong latitudinal gradients of solar heating in the tropical upper stratosphere and lower mesosphere. However, the observed zonal and monthly mean temperature does not follow the solar heating pattern and basically has no latitudinal gradient at and near the equator. As noted by Dunkerton (1989), this indicates that gradient-wind balance holds across the equator. The balance constraint keeps the temperature below radiative equilibrium in the summer hemisphere and above it in the winter hemisphere. The resulting diabatic heating (cooling) is balanced by adiabatic cooling (heating) due to vertical motion associated with a single-cell angular-momentum-conserving Hadley circulation.

The Hadley circulation affects the distribution of the diabatic heating that drives it. Mass conservation requires that streamlines cross from the region of heating to the region of cooling. This gives rise to cross-equatorial advection of absolute angular momentum into the winter hemisphere. As a result, easterlies develop in the equatorial region and westerlies are enhanced at higher latitudes in the winter hemisphere. As described below, using the theory of Held and Hou (1980) as extended by Dunkerton (1989), this leads to a shift in the diabatic heating distribution toward the winter pole.

Analysis of the Hadley circulation is simplified if it is bounded by a lid and no wave drag is present. In the steady-state limit absolute angular momentum is homogenized, deforming the zonal wind into a parabolic profile in sine latitude [see (2.9b) and Fig. 8 in Dunkerton 1989]. The behavior of the vertically integrated temperature can be inferred from the zonal-wind profile at the lid using the thermal-wind relation

$$
\left[f \bar{u}+\bar{u}^{2} \frac{\tan \phi}{a}\right]_{0}^{z_{T}}=-\frac{R}{a H} \frac{\partial}{\partial \phi} \int_{0}^{z_{T}} \bar{T} d z,
$$

where $z_{T}$ is the level of the lid and it is assumed that $\bar{u}=0$ at $z=0$. It is evident that the vertically integrated temperature develops a minimum at the equator in response to tropical easterlies. The vertically integrated deviation of the temperature from radiative equilibrium satisfies an equal-area rule if the diabatic heating is approximated by Newtonian relaxation. The equal-area rule and (7) imply that the temperature minimum at the equator is below radiative equilibrium (see Dunkerton 1989, Fig. 6). Hence, the contour separating the regions 
of diabatic heating and cooling lies in the winter hemisphere and the Hadley circulation is offset toward the winter pole. Another implication is that the tropical easterlies at the lid level reflect the breadth and magnitude of the Hadley circulation. As equatorial easterlies are increased, the vertically integrated temperature becomes progressively colder than radiative equilibrium at the equator and warmer in the winter hemisphere subtropics. Coupled with this is an increase in the latitudinal span of the equatorial cold region and the subtropical warm region [as seen by varying the parameter $\mu_{0}$ in (2.14) of Dunkerton 1989].

Similar arguments to those above can be applied to the case where the domain is deeper than the Hadley circulation and where there is nonmonotonic height dependence in the diabatic heating. Equation (7) applies at any level above $z=0$. In addition, the equal-area rule applies approximately on each level in steady state. This follows from the fact that there can be no net diabatic heating on isentropes in steady state and isentropes are nearly horizontal in the upper stratosphere and mesosphere. So the vertically integrated temperature between $z=0$ and the level of interest approximately satisfies the equal-area rule as well.

\section{b. Behavior of the system in the absence of wave drag}

Here we present results of simulations similar to Dunkerton (1991) for the case with no mechanical forcing, using the balance model described in section 2 . Dunkerton (1989) used a lid at $42 \mathrm{~km}$ to close off the Hadley circulation and produce realistic zonal winds. Extending the domain to include the mesosphere resulted in unrealistically strong equatorial easterlies in the mesosphere (Dunkerton 1989, Fig. 19d). The model used in Dunkerton (1991) had a domain reaching to 84 $\mathrm{km}$ but used a mesospheric Rayleigh damping layer, meant to represent gravity wave drag, to produce a realistic $\bar{m}$ distribution. Our model uses a domain extending from the surface to $100 \mathrm{~km}$ and has no Rayleigh damping in the mesosphere. This latter choice is made to avoid unrealistic feedbacks between the stratosphere and mesosphere arising from the unphysical nature of Rayleigh drag (Shepherd et al. 1996), and is a significant difference from Dunkerton (1991). No attempt is made here to close off the circulation in the stratosphere or mesosphere by mechanical means. We perform several numerical experiments to investigate the effect of inertial adjustment as well as the impact of the choice of diabatic heating representation.

In the first experiment the diabatic heating term $Q$ is approximated by Newtonian cooling with a constant damping rate of $0.1 \mathrm{day}^{-1}$ to a realistic time-dependent radiative equilibrium temperature. The radiative equilibrium temperature was dynamically determined (e.g., Fels 1987) with a comprehensive middle-atmosphere radiative transfer scheme by integrating the thermody-

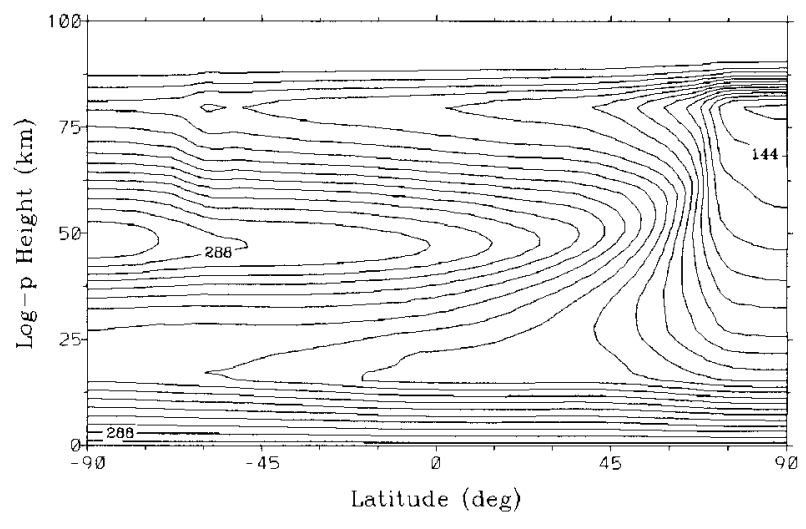

FIG. 1. Solstice $\bar{T}_{\text {rad }}$ for Jan. Contour interval is $8 \mathrm{~K}$.

namic equation without the advection terms for three years. The radiative transfer scheme is based on the middle-atmosphere part of the scheme described by Fomichev and Blanchet (1995). This part of the scheme is valid only above $16 \mathrm{~km}$ and as implemented here uses zonally averaged climatological distributions of ozone and other radiatively active gases. The derived $\bar{T}_{\text {rad }}$ was extended below $16 \mathrm{~km}$ by interpolation with a simple height-dependent temperature profile. Above $80 \mathrm{~km}$ there are large equatorial gradients of $\bar{T}_{\text {rad }}$ and hence thermal driving of a distinct Hadley circulation. As a result, strong cross-equatorial flow develops near the model lid, which can lead to numerical instability. Since the focus here is on the middle atmosphere, the thermal driving above $80 \mathrm{~km}$ is removed by interpolating $\bar{T}_{\text {rad }}$ to a uniform value of $240 \mathrm{~K}$ (see Fig. 1).

The model state about 3 weeks after the Northern Hemisphere $(\mathrm{NH})$ winter solstice of a seasonal cycle simulation without wave drag is shown in Figs. $2 \mathrm{a}-\mathrm{d}$ [the Southern Hemisphere ( $\mathrm{SH}$ ) winter solstice fields are similar but flipped about the equator]. The lowermost (tropospheric) and uppermost (thermospheric) parts of the model domain are not shown since they are regions where $\bar{T}_{\text {rad }}$ is artificially constructed. During equinox seasons, the single-cell Hadley circulation dies away since the latitudinal gradient of the radiative equilibrium temperature vanishes at the equator. Steady-state simulations indicate that $\bar{T}_{\text {rad }}$ has sufficient latitudinal curvature to develop a weak two-cell Hadley circulation at this time, in agreement with the theory of Held and Hou (1980). In fact, the latitudinal curvature reverses sign in the lower tropical stratosphere so that the circulation has a Hadley component overlying an anti-Hadley component. However, during the seasonal cycle simulation this two-cell Hadley circulation is obscured by the transient circulation associated with the seasonal transition. The spring equinox streamfunction from the seasonal integration and from an integration to steady state are shown in Fig. 3.

The solstitial fields shown in Fig. 2 are near steady state, but some transience associated with the seasonal cycle is still present. This is especially evident from the 


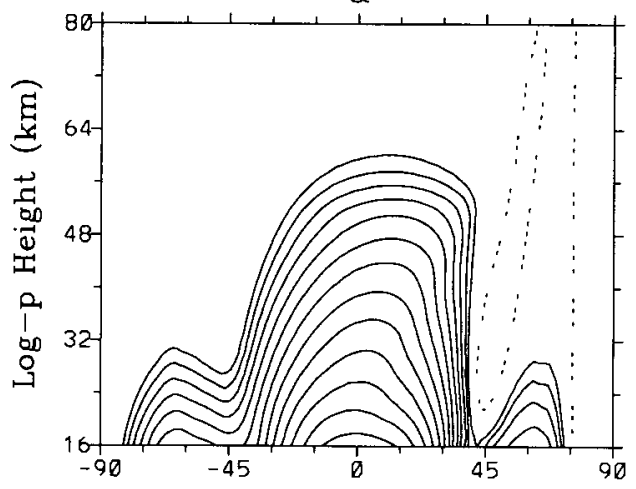

$\mathrm{C}$

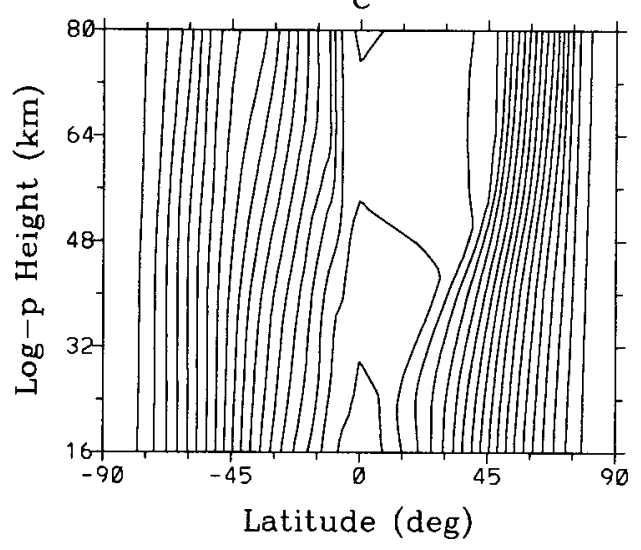

b

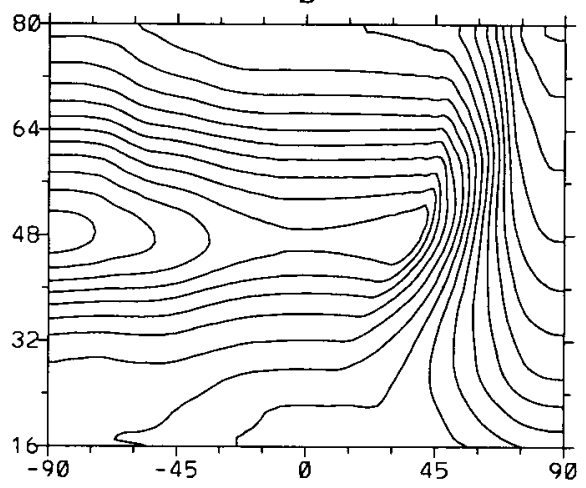

d

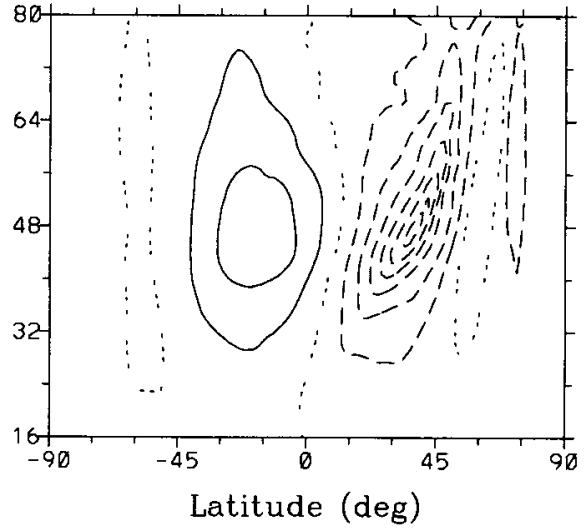

FIG. 2. Results from the annual cycle of the balance model simulation for Jan with a constant Newtonian cooling timescale of 10 days: (a) streamfunction (varying contour intervals specified by $120 \exp (5 x-5)$ $\mathrm{kg} \mathrm{m}^{-1} \mathrm{~s}^{-1}$, where $x=n / 15$ for $\left.0 \leq n \leq 15\right)$; (b) temperature $(8 \mathrm{~K})$; (c) absolute angular momentum (1.5 $\left.\times 10^{8} \mathrm{~m}^{2} \mathrm{~s}^{-1}\right)$; (d) diabatic heating $\left(4.5 \times 10^{-6} \mathrm{~K} \mathrm{~s}^{-1}\right)$. The flow is northward across the equator.

streamlines at lower levels of the stratosphere, which cross $\bar{m}$ isopleths and extend to midlatitudes. This behavior does not persist in the simulations run to steady state with fixed solstitial $\bar{T}_{\text {rad }}$ (not shown). In the stratopause region, the number of overturned $\bar{m}$ isopleths is smaller in the seasonal cycle simulation than in sim- ulations run to steady state, indicative of incomplete $\bar{m}$ homogenization in the former. The latitudinal profile of $\bar{u}$ (Fig. 4a), which approaches an absolute-angularmomentum conserving parabola on the winter side of the equator, is not due to the steady-state limit of the Hadley circulation but rather to inertial adjustment. a

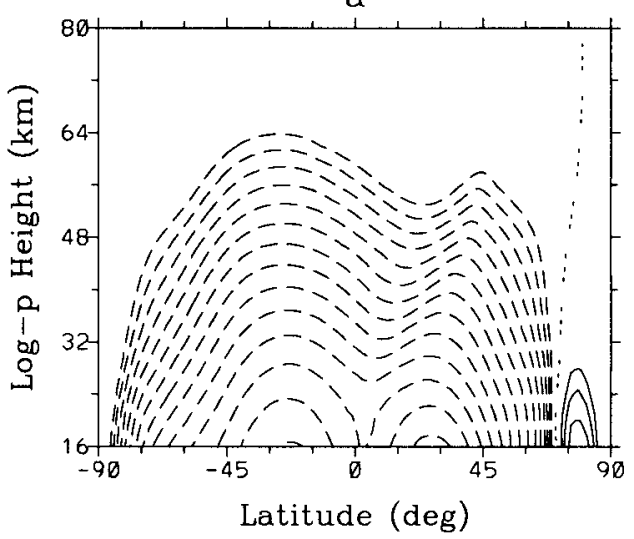

b

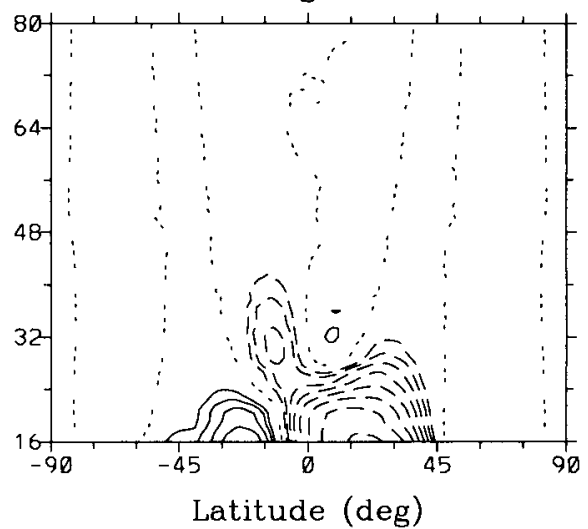

FIG. 3. Equinox $\psi$ for Apr for (a) seasonal cycle run and (b) steady-state run. Contour interval as in Fig. 
a

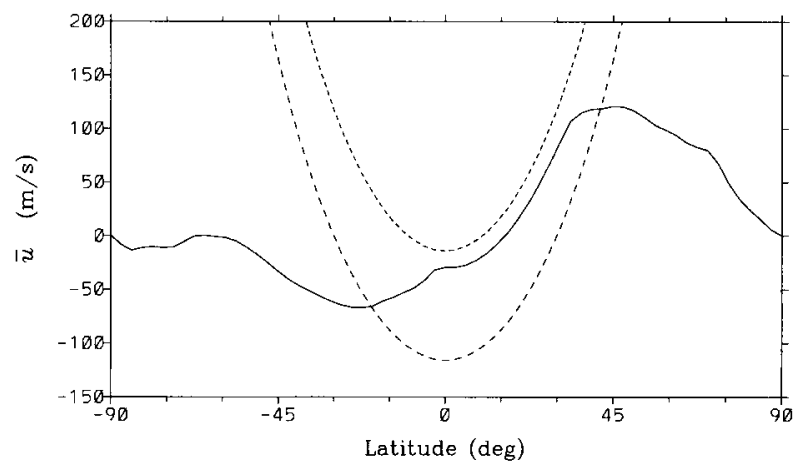

b

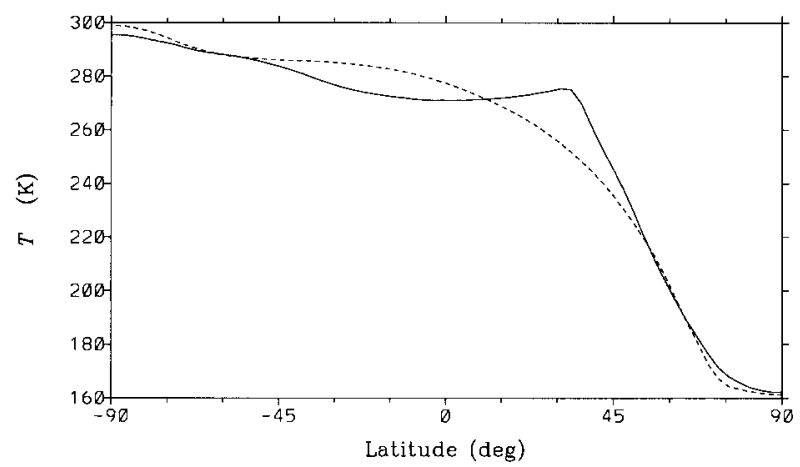

FIG. 4. (a) Latitudinal profile of balance model zonal wind (solid) at $z=45 \mathrm{~km}$ compared with the angular-momentum-conserving parabolas corresponding to $10^{\circ}$ (long dash) and $30^{\circ}$ (dash). (b) Latitudinal profile of $\bar{T}$ (solid) and $\bar{T}_{\text {rad }}$ (dash) at the same level as (a).

In the tropical stratosphere, isopleths of $\bar{m}$ (Fig. 2c) are increasingly deformed with altitude. This is due to the increasing $\bar{v}^{*}$ with height, which advects $\bar{m}$ toward the winter pole and which reflects the vertical structure of the diabatic heating associated with the solar heating gradient. The diabatic heating peaks near the tropical stratopause (Fig. 2d), and diminishes above the stratopause as the solar heating gradient decreases. The latitudinal span of the diabatic heating reveals the extent to which the Hadley circulation spreads beyond the nearequatorial region of divergent $\bar{u}_{\text {rad }}$. Radiative equilibrium is certainly attainable in the subtropics and midlatitudes, yet persistent diabatic heating is found at these latitudes.

The advection of the $\bar{m}$ maximum off the equator triggers inertial adjustment primarily above the stratopause, producing a region of nearly homogenized $\bar{m}$ on the winter side of the equator extending to midlatitudes. This region corresponds to the location of the largest Hadley cell $\bar{v}^{*}$ values and most active inertial adjustment. Based on the model results and simpler tests it is evident that inertial adjustment is always confined within the region of nonzero $\bar{v}^{*}$. As explained below, adjustment actually confines $\bar{v}^{*}$ closer to the equator. So it is not possible for adjustment to extend the Hadley circulation to higher latitudes.
Analysis of the model fields shows that the direct contribution of inertial adjustment to the diabatic heating at every time step is small. It is most noticeable at the winter hemisphere edge of the homogenized $\bar{m}$ region and on the summer side of the equator. These are the regions where the latitudinal gradient of $\bar{m}$ is largest and there is the greatest poleward advection of angular momentum. The zonal wind maximum and sharp latitudinal gradient in the temperature profile (Fig. 4b) develop at the poleward edge of the adjustment region through the poleward transfer of angular momentum by $\bar{v}^{*}$. Inertial adjustment differs from the effect of $\bar{v}^{*}$ in that it transports angular momentum equatorward instead of toward the winter pole. Thus, it acts to limit the magnitude of the easterlies in the Tropics. The latitudinal profile of the zonal wind shows that the effect of inertial adjustment is significant. From (7) and the equal-area rule it can then be inferred that the vertically integrated diabatic heating is significantly modified. The numerical steady-state solution obtained with inertial adjustment by Dunkerton (1989) (see his Fig. 9) demonstrates this effect: his solution exhibited a significantly weaker diabatic heating dipole compared to that obtained with the parabolic approximation but centered at the same latitude.

The solstitial Hadley circulation (Fig. 2a) progressively shifts toward the winter pole with altitude. This deformation is tied to the increasing deflection of $\bar{m}$ isopleths toward the winter pole with altitude, and the corresponding shift in the diabatic heating dipole off the equator. In the lowest part of the tropical stratosphere, however, $\bar{m}$ remains close to its planetary value and the diabatic heating is centered on the equator together with the Hadley circulation. The tendency for streamline alignment with $\bar{m}$ contours arises from the same constraint on evolution to steady state that leads to the downward control effect (Haynes et al. 1991), namely, that streamlines cannot cross $\bar{m}$ isopleths in steady state in regions without mechanical forcing. The difference is that in the extratropics, $\bar{m}$ isopleths are vertically aligned and have limited mobility so that they organize the streamfunction distribution. In the Tropics, by contrast, the greater mobility of $\bar{m}$ contours becomes an important factor in the evolution to steady state since the streamfunction significantly affects the $\bar{m}$ distribution, forming a nonlinear feedback. Indeed, it is this feature that allows a steady circulation to develop without wave drag or viscosity (Held and Hou 1980).

The results presented here based on a realistic $\bar{T}_{\text {rad }}$ distribution differ from those of Dunkerton (1989) in the mesosphere. Dunkerton found that extending the domain in his model from 42 to $84 \mathrm{~km}$ along with the thermal driving gave unrealistically strong easterlies with a peak magnitude of $-280 \mathrm{~m} \mathrm{~s}^{-1}$ near the equator (see his Fig. 19d). It is not clear what form $\bar{T}_{\text {rad }}$ took in his extended model, but it contributed to these excessive easterlies. The absence of explicit inertial adjustment 


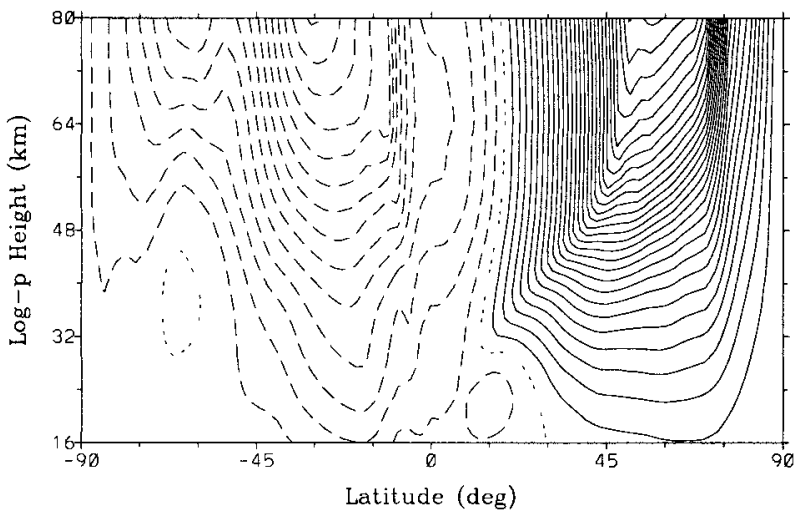

FIG. 5. Model $\bar{u}$ corresponding to Fig. 2c. Contour interval is $10 \mathrm{~m}$ $\mathrm{s}^{-1}$.

also contributed since the positive latitudinal shear in the zonal wind at the equator coupled with positive $\bar{v}^{*}$ in the mesosphere combine to produce significant advection of easterly momentum into the Tropics. This advection vanishes in steady state, but inertial adjustment acts like a short circuit that limits the magnitude of the easterlies. Dunkerton (1991) used a $\bar{T}_{\text {rad }}$ with more realistic characteristics, but its impact was obscured by the use of a friction layer in the mesosphere to represent gravity wave drag. In the model used here the easterlies did not exceed a value of $-135 \mathrm{~m} \mathrm{~s}^{-1}$ (for the radiative damping rate used) and formed a subtropical jet in the summer hemisphere (Fig. 5).

In further experiments, the limitations of simple Newtonian cooling are explored. In particular, we look at the influence of the choice of the vertical profile of the radiative relaxation rate on the structure of the circulation and $\bar{m}$. The effect of using a comprehensive radiative transfer scheme on the solutions is also considered.

The overturning of $\bar{m}$ contours in the tropical stratopause region of the balance model is due to the strong cross-equatorial flow produced by the Hadley circulation diabatic heating dipole, which happens to maximize at these levels. This feature is suggestive of the pattern seen in observations (Fig. 6) and GCM simulations (Fig. 19) below the mesosphere. However, the assumption of a uniform radiative damping rate used in the simulation above is unrealistic given the generally decreasing optical thickness of the atmosphere with altitude. Yet if the constant radiative damping rate is naively replaced with a monotonically increasing function of height then the diabatic heating and the region of horizontal $\bar{m}$ contours in the model shift upward in an unrealistic manner (Fig. 7). The function used is

$$
\left[78 \exp \left(-\frac{z-16}{12}\right)+2\right]^{-1} \text { day }^{-1},
$$

where $16 \mathrm{~km}<z<80 \mathrm{~km}$, which crudely approximates

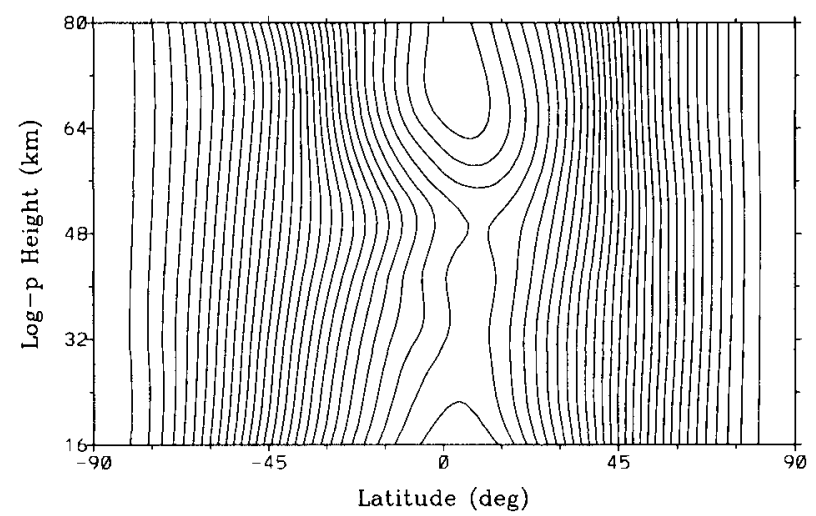

FIG. 6. CIRA Jan mean $\bar{m}\left(10^{8} \mathrm{~m}^{2} \mathrm{~s}^{-1}\right)$.

the vertical variation of the damping rate over the depth of the middle atmosphere.

This behavior can be explained by the fact that the balance constraint on the latitudinal temperature profile is insensitive to the damping rate (except possibly in the upper mesosphere where the damping timescale is on the order of a day): the deviation of $\bar{T}$ from $\bar{T}_{\text {rad }}$ depends only on the latitudinal profile of $\bar{T}_{\text {rad }}$. It follows that the amplitude of the diabatic heating is affected primarily by the radiative damping rate. Thus, the radiative damping rate acts as a weighting function which, in the monotonically increasing case, shifts the heating to higher altitudes even though the latitudinal gradient in the radiative equilibrium temperature is weaker there.

In fact, the radiative damping rate is sensitive to temperature. The damping rate increases in the upper stratosphere, maximizing near the stratopause (Andrews et al. 1987), reflecting the vertical profile of the radiative equilibrium temperature. Consequently, the region of largest solar heating gradients also corresponds to a local maximum in the damping rates. The balance model simulation was therefore repeated using the comprehensive radiative transfer scheme of Fomichev and Blanchet (1995) instead of Newtonian cooling (Fig. 8). The diabatic heating dipole (Fig. 8d) is stronger compared to the one in the first experiment (Fig. 2d), reflecting the shorter radiative damping timescale and its dependence on the local temperature. The diabatic heating is also confined closer to the stratopause. This feature manifests itself in the streamfunction (Fig. 8a) as more concentrated and horizontal streamlines just above the stratopause. In the lower tropical stratosphere the streamfunction is weaker since the radiative damping time is much longer than 10 days.

To summarize the results of this subsection, the inclusion of a more realistic inertial adjustment scheme and extension of the domain into the mesosphere (without Rayleigh drag) has yielded additional insight into the Hadley circulation. The circulation penetrates to midlatitudes in the mesosphere. The significant redistribution of the zonal wind and temperature by inertial adjustment in the upper stratosphere and mesosphere 


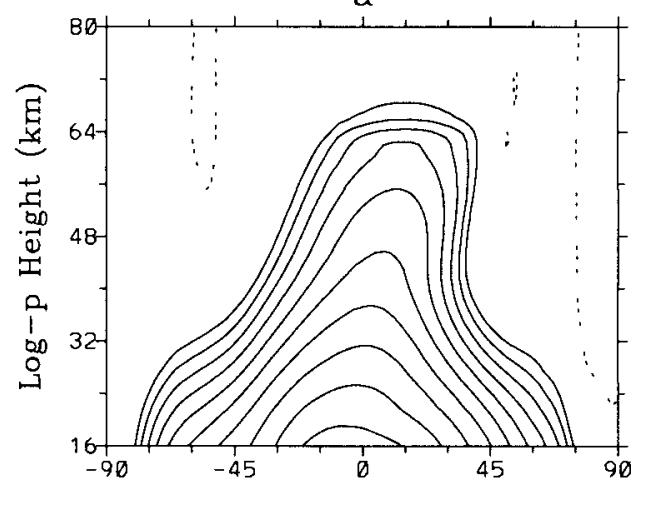

$\mathrm{C}$

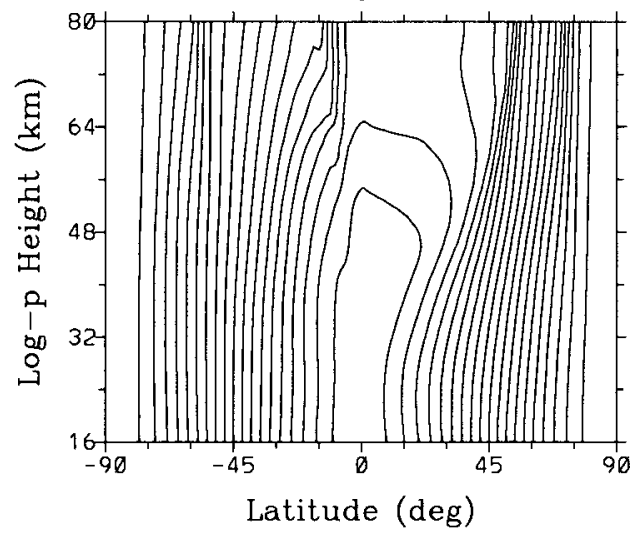

$\mathrm{b}$
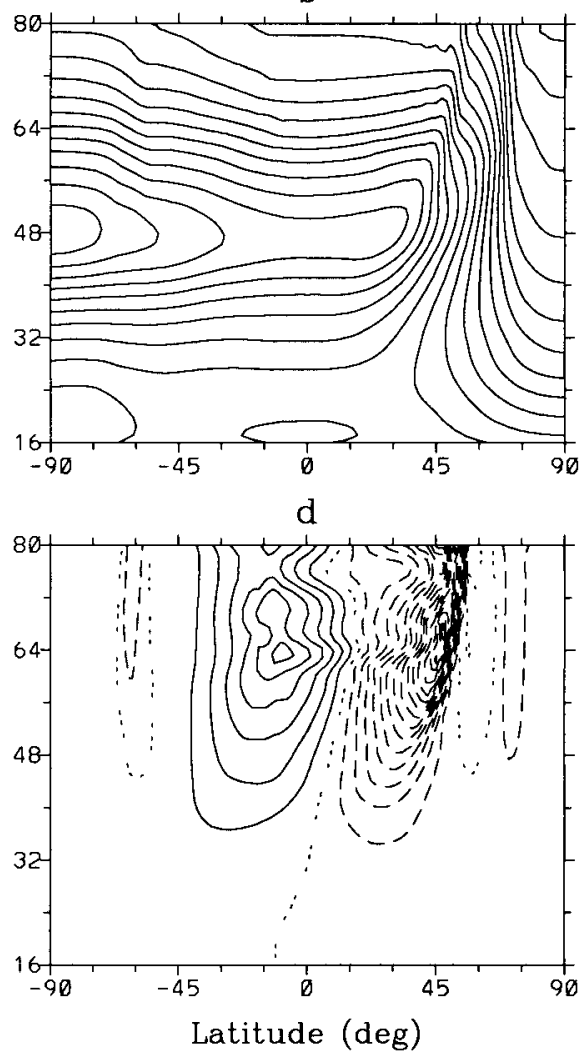

FIG. 7. Same as Fig. 2 but for the balance model simulation with a Newtonian cooling rate monotonically increasing with height.

limits the strength of the Hadley circulation but does not have much of an effect on its latitudinal extent. The overturning of $\bar{m}$ contours in the stratopause region is a feature determined by the vertical distribution of radiative heating and radiative damping. It appears that the excessive equatorial easterlies in the mesosphere of the model of Dunkerton (1989) are an artifact of the radiative driving used, but the lack of explicit inertial adjustment in that model also played a role. In our model the Hadley circulation closes off gradually in the mesosphere.

\section{c. Role of tropical wave drag}

So far wave drag has been neglected, but it is an important element in the formation of the middle-atmosphere zonal wind and temperature distributions. The model $\bar{m}$ field shown in Fig. 2 departs significantly from the observed one (Fig. 6) above the stratopause; in the real atmosphere the region of horizontal $\bar{m}$ contours is confined to the stratopause and lower mesosphere region. The degree of contour deflection into the winter hemisphere in the stratosphere is also smaller.

Dunkerton (1991) used a relaxational damping layer in the mesosphere to represent the action of wave drag and produce a qualitatively correct distribution of $\bar{m}$. This relaxational drag acts to restore the vertical orientation of $\bar{m}$ isopleths in the mesosphere by pulling the zonal wind toward zero. Plumb and Eluszkiewicz (1999) have also noted the stabilizing effect of weak relaxational damping on the $\bar{m}$ distribution of the tropical stratosphere. However, the momentum flux available for wave drag is fixed, aside from variations in tropospheric sources. Zonal wind changes can only redistribute this fixed amount of wave drag. In contrast, a relaxational damping layer is an infinite reservoir for local momentum dissipation (Shepherd et al. 1996). Tropical wave drag can also accelerate the zonal wind rather than damp it, as manifested by the QBO and SAO.

Nevertheless, relaxational damping imitates some types of wave drag in the Tropics that pull the zonal wind toward zero and counteract the development of very strong tropical easterlies. This is suggested by the restoration of vertical $\bar{m}$ isopleths in the observed tropical mesosphere (Fig. 6) even in the presence of strong cross-equatorial flow. Observations also indicate that the deformation of $\bar{m}$ isopleths in the subtropics of the model upper stratosphere is too great. There is evidence that this region is subject to significant stationary planetarywave breaking, which damps westerlies. In general, subtropical and midlatitude wave drag acts to reduce the 


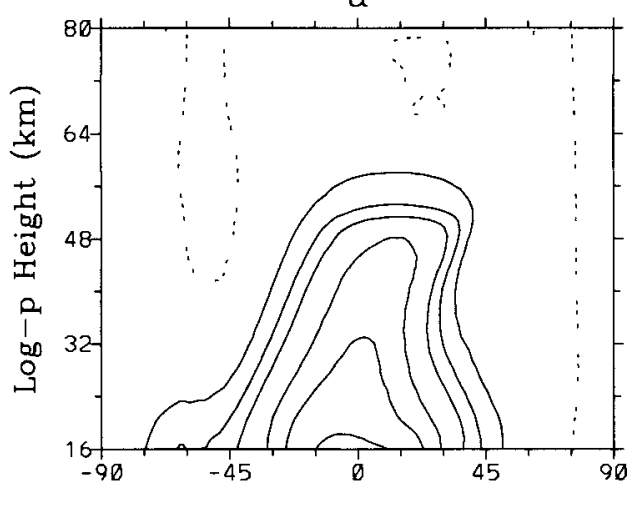

$\mathrm{C}$

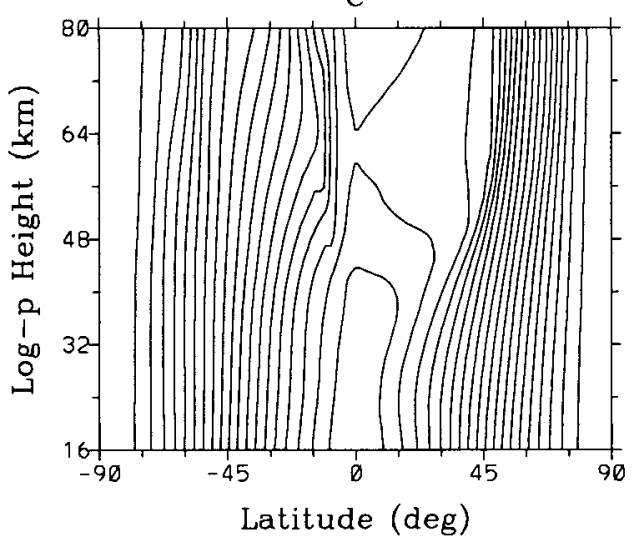

b

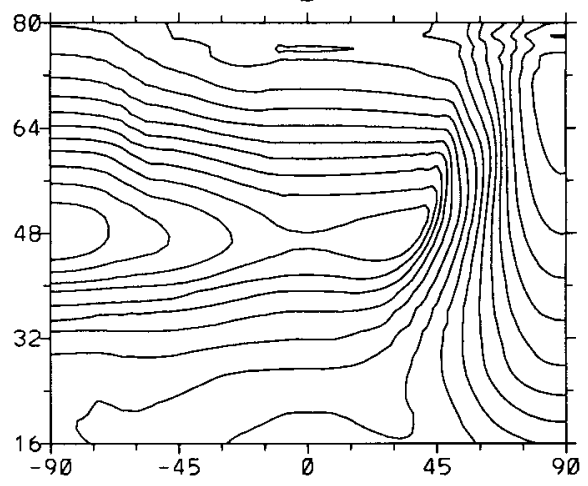

d

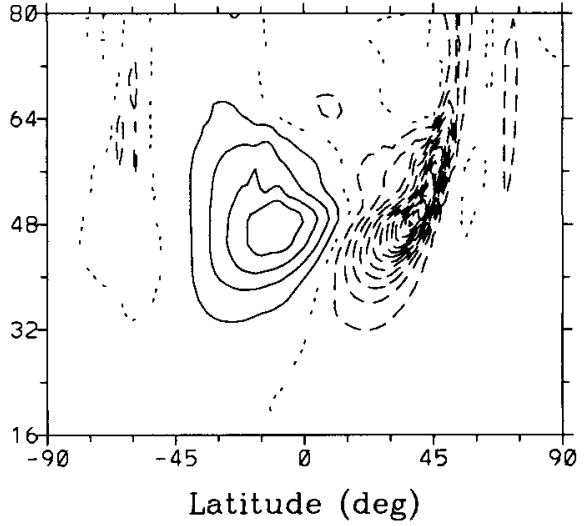

FIG. 8. Same as Fig. 2 but for the balance model simulation using a comprehensive radiative transfer scheme.

magnitude of the zonal flow in the stratosphere and mesosphere of the winter hemisphere. So the large region of $\bar{m}$ homogenization seen in the model can be expected to be confined closer to the equator, if not removed completely. This issue is pursued further in section 4.

\section{d. Validity of the balance model}

A significant physical assumption underlying the results presented above is gradient-wind balance. However, the latter is an empirically motivated approximation and not an instantaneous constraint that is satisfied by the real atmosphere, or indeed by more comprehensive models that resolve wave and instability processes. We therefore use a mechanistic model based on the three-dimensional primitive equations (Saravanan 1992) to test the validity of the balance model simulations. The same latitude-height domain and Newtonian cooling scheme are used in both models. The models have the same vertical diffusion but different horizontal diffusion, but this is not considered to be important for the basic structure of the solutions. Both models were run for 91 days to mid-July from a state of rest, with a gradual ramping in the first month to a seasonally evolving radiative equilibrium temperature. This integration period is much longer than the inertial adjustment timescale in the three-dimensional model (a few days) and is sufficient to establish the pattern of evolution of the slow component. Comparing the zonal-mean states at the end of the simulation period (Fig. 9), it is evident that they have a high degree of similarity on large scales, although there are large-amplitude small-scale disturbances in the primitive equations model state. These disturbances occur in the same inertially unstable region as found in the two-dimensional balance model, and are evidence of explicitly resolved inertial instability.

Features of inertial instability are obscured by zonal averaging of the longitudinally dependent model fields shown here. The relative weakness of the disturbances in the zonal mean compared to zonally symmetric simulations (Saravanan 1992) suggests that the zonally asymmetric component is more prominent. This is consistent with the stability analysis of Dunkerton (1983), which predicts zonally asymmetric modes of inertial instability as being the fastest growing. Nevertheless, zonally symmetric modes are not negligible and are active in the winter hemisphere adjustment as can be seen from the zonal mean fields (see also Clark and Haynes 1996).

Note the apparent insensitivity of the Hadley circulation to the details of the inertial adjustment process. 

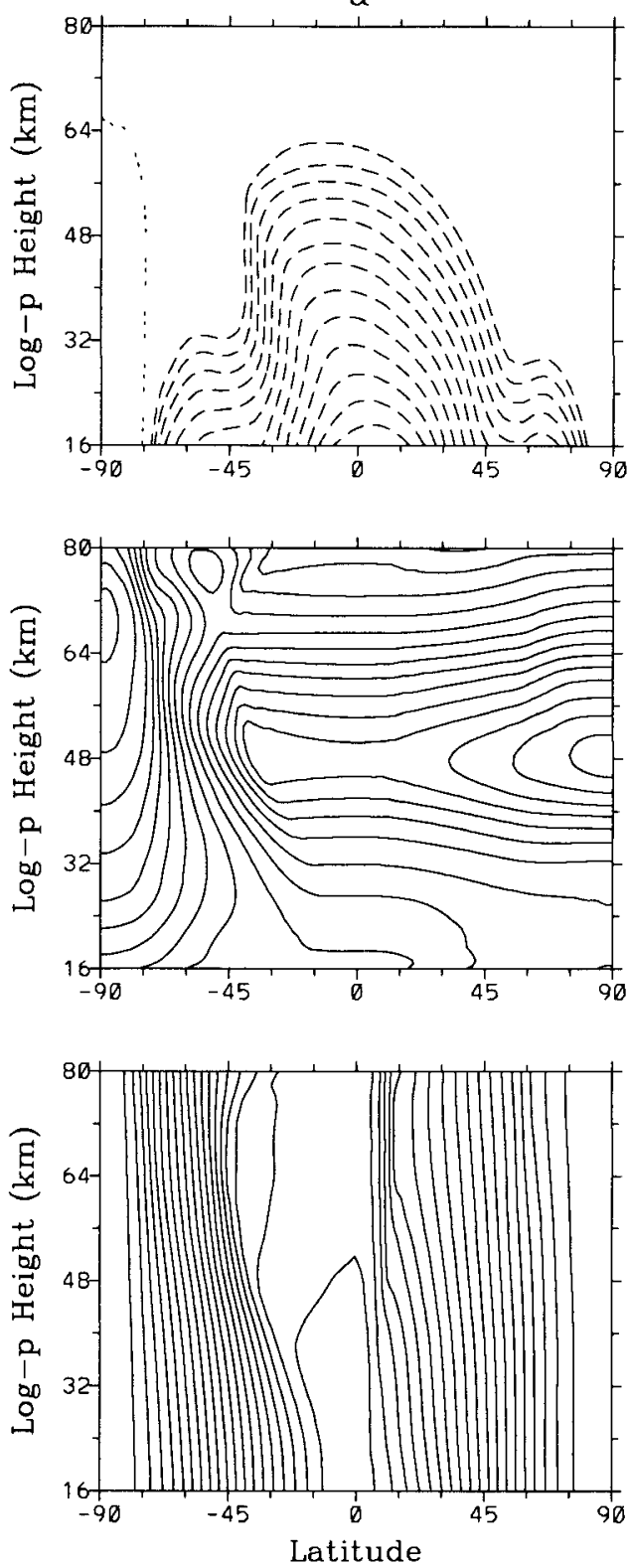
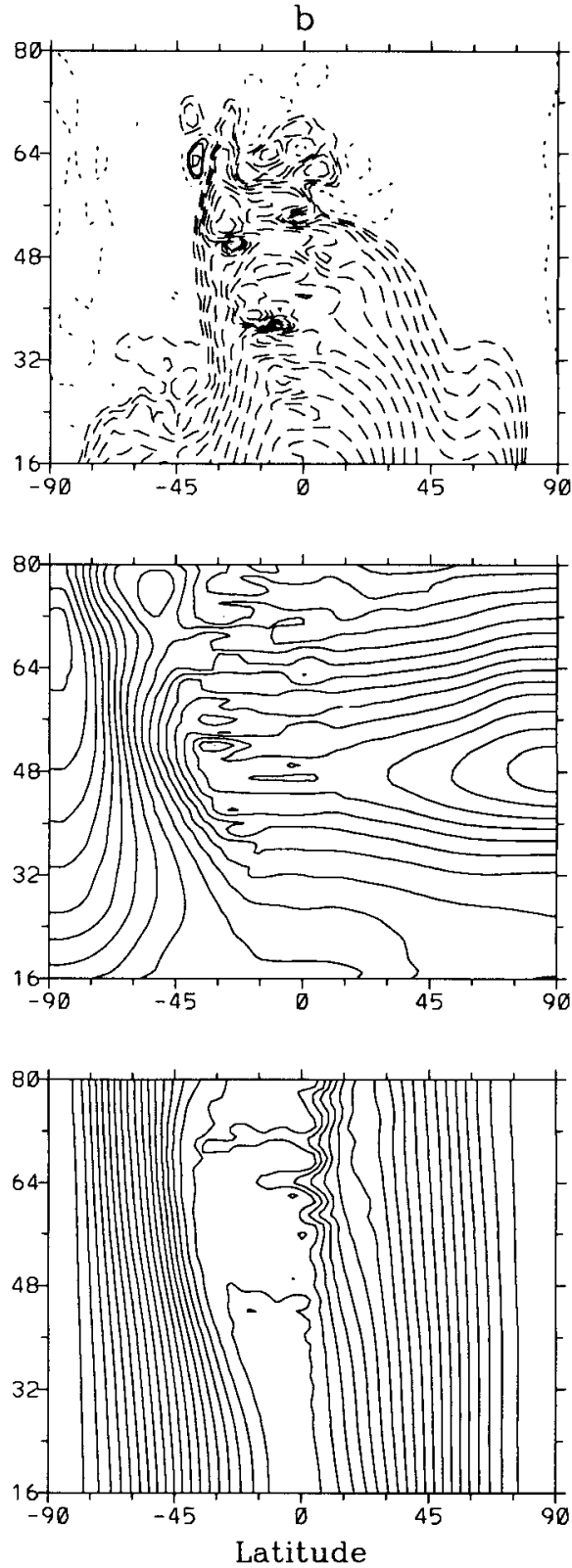

FIG. 9. (a) Balance model $\psi, \bar{T}$, and $\bar{m}$ fields in Jul. Contour intervals as in Fig. 2. (b) Same fields as (a) for a $3 \mathrm{D}$ primitive equations model with a resolution set to T42 and 60 levels.

The overall form and amplitude of this circulation in the primitive equations model matches that of the balance model, except for the additional direct contribution from inertial instability, which is largest in the winter hemisphere mesosphere. The difference of the left and right panels in Fig. 9 (not shown) indicates that the small-scale disturbances are essentially superimposed on the large-scale fields and that the amplitude of the circulation is nearly identical in the two models. On the basis of this simulation, it appears that gradient-wind imbalance has only a local effect. It also appears that the balance model inertial adjustment scheme is re- sponding correctly to the distribution of the zonal wind, considering the $\bar{m}$ homogenization in the primitive equations model.

\section{Influence of the wave-driven circulation on the Hadley circulation}

A feature of the nonlinear balance system is the lack of self-advection in the meridional circulation. On account of gradient-wind and hydrostatic balance, the inertial terms, namely, $D \bar{v}^{*} / D t$ and $D \bar{w}^{*} / D t$, are neglected in the meridional and vertical momentum equations. The 
inertial terms contain the nonlinear (self-advection) and tendency parts of these equations. Neglect of the tendency terms makes the meridional circulation an instantaneous, nonlocal field that requires the presence of mechanical or diabatic forcing to exist. Neglect of the advection terms makes the streamfunction equation linear. (A streamfunction equation can be derived even if balance is not assumed, but it is nonlinear and contains time derivatives.) Thus, advective nonlinearity in the balance system manifests itself only as redistribution of the $\bar{m}$ and $\bar{\theta}$ fields (equivalently, $\bar{u}$ and $\bar{T}$ ), which can modify $\psi$ through $Q \mathcal{F}$, and the coefficients of the operator $\mathcal{L}$.

In general, the wave drag $\mathcal{F}$ and the diabatic heating $Q$ are nonlinear, nonlocal functions of the zonal wind and temperature, respectively. Here we assume that wave drag is a prescribed field. For the diabatic heating the linear Newtonian relaxation approximation is used with a uniform damping rate of $0.1 \mathrm{day}^{-1}$. These assumptions simplify the problem but leave enough to investigate the basic zonal-mean interaction of the Hadley circulation and subtropical wave drag. The tropical region is defined here as extending between $20^{\circ} \mathrm{S}$ and $20^{\circ} \mathrm{N}$, based roughly on the subtropical edge of the surf zone in the lower stratosphere (above 16 $\mathrm{km})$. The influence of the Hadley circulation on the subtropical wave drag distribution is best dealt with in a more comprehensive model that can resolve waves and wave breaking, so will not be addressed here. The effect of the Hadley circulation on the wave-driven circulation (WDC) is considered in Semeniuk and Shepherd (2001).

To a good approximation, extratropical zonal-mean dynamics are linear due to the stable stratification of $\bar{m}$ in latitude and $\bar{\theta}$ in altitude. Specifically,

$$
\frac{1}{a} \frac{\partial \bar{m}}{\partial \phi} \sim a \cos \phi f \quad \text { and } \quad\left|\frac{1}{a} \frac{\partial \bar{m}}{\partial \phi}\right| \gg\left|\frac{\partial \bar{m}}{\partial z}\right|
$$

while

$$
\frac{\partial \bar{\theta}}{\partial z} \sim e^{\kappa z / H} S_{0} \text { and } \quad\left|\frac{\partial \bar{\theta}}{\partial z}\right| \gg\left|\frac{1}{a} \frac{\partial \bar{\theta}}{\partial \phi}\right|
$$

(where $S_{0}$ is the static stability for an isothermal atmosphere with $\bar{T}=240 \mathrm{~K}$, although $S_{0}$ based on a standard reference $\bar{T}$ profile is more accurate). As a result, to leading order the absolute angular momentum equation is

$$
\frac{\partial \bar{m}}{\partial t}-a \cos \phi f \bar{v}^{*}=a \cos \phi \mathcal{F}, \quad \text { i.e., } \frac{\partial \bar{u}}{\partial t}-f \bar{v}^{*}=\mathcal{F},
$$

and the thermodynamic equation is

$$
\frac{\partial \bar{\theta}}{\partial t}+e^{\kappa z / H} S_{0} \bar{w}^{*}=e^{\kappa z / H} Q, \quad \text { i.e., } \frac{\partial \bar{T}}{\partial t}+S_{0} \bar{w}^{*}=Q .
$$

Given specified $\mathcal{F}$ and assuming Newtonian cooling
$Q=-\alpha\left(\bar{T}-\bar{T}_{\mathrm{rad}}\right)$, the linear Hadley circulation satisfies

$$
\begin{aligned}
& \frac{\partial \bar{u}_{H}}{\partial t}-f \bar{v}_{H}^{*}=0, \\
& \frac{\partial \bar{T}_{H}}{\partial t}+S_{0} \bar{w}_{H}^{*}=-\alpha\left(\bar{T}_{H}-\bar{T}_{\mathrm{rad}}\right),
\end{aligned}
$$

as well as the continuity and (linear) thermal-wind equations, while the linear wave-driven circulation satisfies

$$
\begin{gathered}
\frac{\partial \bar{u}_{F}}{\partial t}-f \overline{\boldsymbol{v}}_{F}^{*}=\mathcal{F}, \\
\frac{\partial \bar{T}_{F}}{\partial t}+S_{0} \bar{w}_{F}^{*}=-\alpha \bar{T}_{F} .
\end{gathered}
$$

These component solutions for the wave-driven and Hadley circulations are independent and satisfy the full equations when added together:

$$
\begin{aligned}
& \frac{\partial\left(\bar{u}_{H}+\bar{u}_{F}\right)}{\partial t}-f\left(\bar{v}_{H}^{*}+\bar{v}_{F}^{*}\right)=\mathcal{F} \\
& \frac{\partial\left(\bar{T}_{H}+\bar{T}_{F}\right)}{\partial t}+S_{0}\left(\bar{w}_{H}^{*}+\bar{w}_{F}^{*}\right)=-\alpha\left(\bar{T}_{H}+\bar{T}_{F}-\bar{T}_{\mathrm{rad}}\right)
\end{aligned}
$$

The linear Hadley circulation has certain unphysical characteristics, which are described in Semeniuk and Shepherd (2001). However, the above serves to demonstrate that the Hadley circulation and the wave-driven circulation (for specified $\mathcal{F}$ and Newtonian cooling) interact only through the advection of $\bar{m}$ and $\bar{\theta}$.

In the Tropics the Coriolis term $f$ is small so the advective terms in the zonal-wind equation are relatively more important and cannot be neglected (i.e., $a^{-1} \partial \bar{m} / \partial \phi$ can deviate from $-a \cos \phi f$ significantly). In contrast to the zonal-wind equation, the thermodynamic equation remains linear to leading order in the Tropics since $\bar{\theta}$ is stably stratified at low latitudes.

The Tropics are characterized by the breakdown of ellipticity in the streamfunction operator $\mathcal{L}$. In the linear system, nonellipticity is confined to the equator itself where $\mathcal{L}$ becomes parabolic. In the nonlinear system, the nonelliptic behavior can occur over a broad span of tropical latitudes during solstice seasons, as shown in section 3 . In either case, the tropical $\bar{w}^{*}$ distribution produced by mechanical forcing in the subtropics can have no meridional gradient at the equator. It also cannot have an excessive meridional curvature. This follows from the fact that thermal-wind balance is built into the streamfunction equation. Hence, it is not possible for subtropical wave drag to produce a tropical temperature distribution that would support a Hadley circulation of either the Dunkerton (1989) or the Held and Hou (1980) variety. By the same token, subtropical wave drag also cannot entirely suppress the Hadley circulation.

The purpose here is therefore to investigate how the 


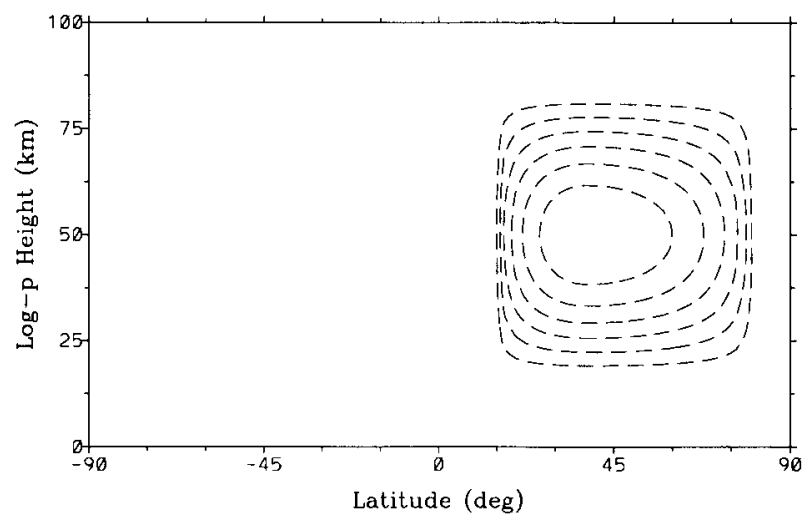

FIG. 10. Model $\mathcal{F}$. Contour interval $1 \mathrm{~m} \mathrm{~s}^{-1} \mathrm{day}^{-1}$.

WDC-induced redistribution of $\bar{m}$ (and $\bar{\theta}$ ) in the Tropics modifies the Hadley circulation. We conduct a numerical test involving a prescribed region of mechanical forcing with annual periodicity in the model extratropics. Another approach would be to use a more comprehensive wave drag parameterization (e.g., Garcia 1991), but it is believed that the essential aspects can be captured with an idealized wave drag. The imposed wave drag has the form

$$
\mathcal{F}(\phi, z, t)=F_{0} Y(\phi)^{1 / 5} Z(z)^{1 / 2} H(t),
$$

with $F_{0}=-7 \mathrm{~m} \mathrm{~s}^{-1}$ day $^{-1}$ (Fig. 10). The latitudinal dependence is defined by

$$
Y(\phi)= \begin{cases}\frac{1}{2}-\frac{1}{2} \cos \left(\pi \frac{\phi-\phi_{1}}{\phi_{2}-\phi_{1}}\right) & \text { for } \phi_{1} \leq \phi \leq \phi_{2} \\ \frac{1}{2}+\frac{1}{2} \cos \left(\pi \frac{\phi-\phi_{2}}{\phi_{3}-\phi_{2}}\right) & \text { for } \phi_{2} \leq \phi \leq \phi_{3} \\ 0 & \text { otherwise, }\end{cases}
$$

with $\phi_{1}=15^{\circ}$ being the subtropical limit of the forcing, $\phi_{2}=38^{\circ}$ the latitude of its maximum, and $\phi_{3}=$ $80^{\circ}$ its northern limit. The vertical dependence is defined by

$$
Z(z)= \begin{cases}\frac{1}{2}+\frac{1}{2} \cos \left[2 \pi \frac{z-\frac{1}{2}\left(z_{1}+z_{2}\right)}{z_{2}-z_{1}}\right] & \text { for } z_{1}<z<z_{2} \\ 0 & \text { otherwise, }\end{cases}
$$

with $z_{1}=16 \mathrm{~km}$ the lower limit and $z_{2}=84 \mathrm{~km}$ the upper limit of the forcing region. The time dependence is defined by

$$
H(t)=\left\{\begin{array}{lr}
\sin ^{2}\left(\pi \frac{t+30}{91}\right) & \text { for } 0 \leq t \leq 61 \\
0 & 335 \leq t \leq 365 \\
0 & \text { for } 61 \leq t \leq 335
\end{array}\right.
$$

(with $t$ in days) so that it acts only during the winter season. In order to qualitatively separate the effect of the WDC on $\bar{m}$ alone or in combination with the Hadley circulation, the radiative equilibrium temperature was respectively prescribed to be either isothermal or climatologically derived (as in section 3 ).

In the isothermal $\bar{T}_{\text {rad }}$ case (Fig. 11), where there is no Hadley circulation, the effect of the imposed $\mathcal{F}$ on the $\bar{m}$ distribution in the Tropics is limited. There is no significant isopleth deformation although a strong gradient in $\bar{m}$ forms in the subtropics at the edge of the forcing region. The response in the climatological case (Fig. 12) is quite different, with greater $\bar{m}$ isopleth deformation in the tropical upper stratosphere and mesosphere than is evident in Fig. 2. There is also a noticeable alteration of the diabatic heating that occurs on both sides of the equator and at the stratopause. Part of this, but not all, is a direct result of the forcing, which imposes a quadrupole heating pattern of its own (Fig. 11d).

The $\bar{m}$ deformation in the tropical upper stratosphere and lower mesosphere in the climatological case persists much longer than the duration of the forcing and lasts into the following year. This slow relaxation toward radiative equilibrium in the Tropics is due to the "flywheel effect" (Scott and Haynes 1998) resulting from the decoupling of temperature and zonal wind near the equator. The range of altitudes where the severe $\bar{m}$ isopleth distortion occurs is partly determined by the height of the imposed wave drag maximum (at $50 \mathrm{~km}$ ), which corresponds to the peak cross-equatorial flow and is reflected in the intensification of the diabatic heating. However, it can be seen from Fig. 11c that without the Hadley circulation and inertial adjustment the WDC cannot produce the sort of isopleth distortion seen in Fig. 12c. In the climatological case the tropical state is conditioned in the stratopause region in a way that enhances the cross-equatorial flow associated with the WDC. This effect, also noted by Tung and Kinnersley (2001), is pursued further in Semeniuk and Shepherd (2001).

The $\bar{m}$ deformation in the tropical stratopause region (Fig. 6) is consistent with observational inferences that point to an increase with height in both the equatorward penetration and amplitude of wave drag and hence of the WDC. In the model subtropics, the imposed wave drag acts to partially suppress the advective and inertial adjustment deformation of $\bar{m}$ (cf. Fig. 12c with Fig. 2c), which brings the horizontal $\bar{m}$ stratification closer to that observed.

To what extent can the effects of the WDC and the Hadley circulation be separated? The total circulation exists as an instantaneous reflection of the diabatic heat- 


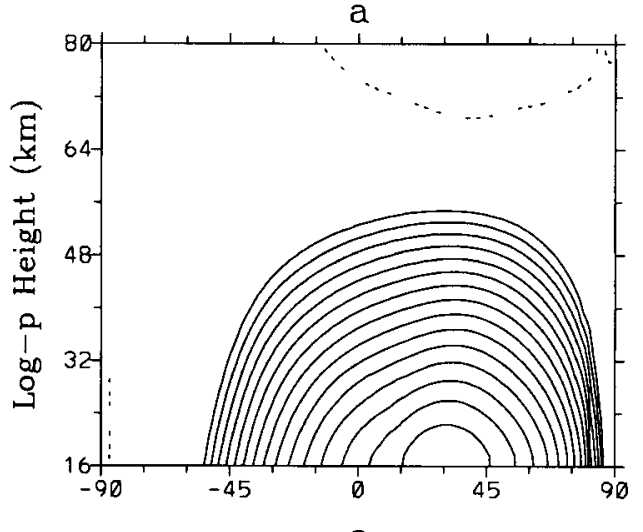

c

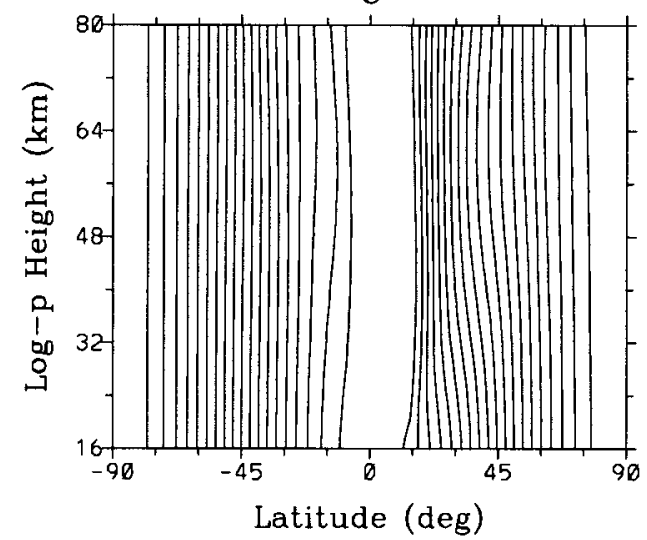

b

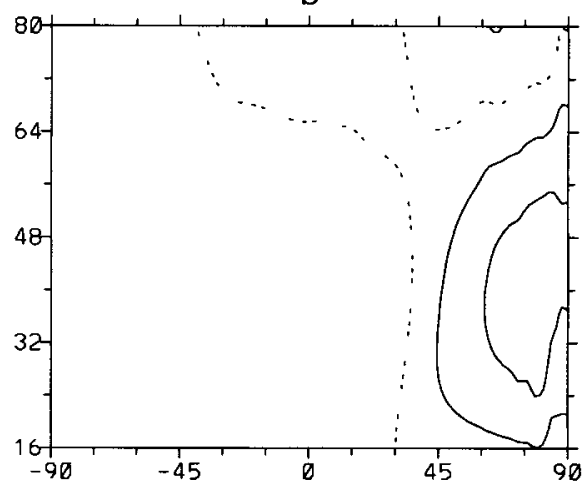

d

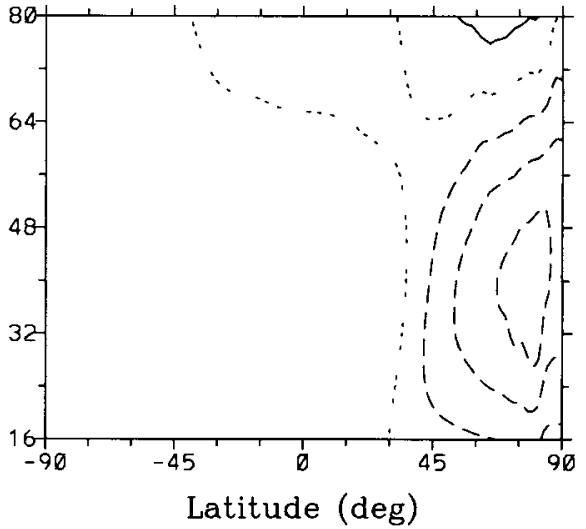

FIG. 11. Jan distribution of (a) $\psi$ (contour levels as in Fig. 2), (b) $\bar{T}(5 \mathrm{~K})$, (c) $\bar{m}\left(1.5 \times 10^{8} \mathrm{~m}^{2} \mathrm{~s}^{-1}\right)$, and (d) $Q\left(4.5 \times 10^{-6} \mathrm{~K} \mathrm{~s}^{-1}\right)$ from the nonlinear balance model with $T_{\text {rad }}=240 \mathrm{~K}$ and forcing specified in the text.

ing and mechanical forcing. If the diabatic heating could be decomposed into the part induced by wave drag and the part associated with the Hadley circulation, then so could the total circulation. However, even if $Q$ is linear in $\bar{T}$ and $\mathcal{F}$ is prescribed, the possibility of a quantitative separation of the two components of the circulation is not clear. There is nonlinear coupling of the two components through the redistribution of $\bar{m}$ and $\bar{T}$, which is reflected in the coefficients of $\mathcal{L}$ and in the diabatic heating.

Although quantitative changes arise in the diabatic heating of the Hadley circulation in the presence of the WDC, qualitatively it remains intact. It was noted previously that the WDC is prevented from producing equatorially asymmetric heating and a latitudinal temperature gradient near the equator. This means that the WDC cannot alter the Hadley circulation in the immediate vicinity of the equator. Figure 13 illustrates this property. The departure of the observed temperature from radiative equilibrium (Fig. 13a) shows strong meridional asymmetry about the equator. But the difference between the observed temperature and the temperature from the balance model simulation with a comprehensive radiative transfer scheme (Fig. 13b), which presumably is due to the WDC, is symmetric about the equator with very little gradient out to $\pm 15^{\circ}$. Thus, it would appear that $\bar{T}$ from the model case without mechanical forcing captures most of the equatorially asymmetric component of the temperature difference in the Tropics.

The difference of the fields in Figs. 11a,d from the corresponding fields in Figs. 12a,d is shown in Fig. 14 and is to be compared with Fig. 2. It is seen that Figs. 2 and 14 are very similar in the Tropics, including the cross-equatorial mass flow, suggesting that the Hadley circulation is, to a first approximation, unaltered by the presence of the WDC. The main differences emerge in the northern middle latitudes, but they reflect as much the effect of the Hadley circulation on the WDC as they do the converse-and, ultimately, the distinction may not be meaningful.

\section{The Hadley circulation in a middle-atmosphere GCM}

The balance model is a reasonable starting point for the study of the Hadley circulation, but the goal is to identify this process in the real atmosphere. Current efforts to understand the atmosphere include GCM studies. Given the limited nature of observational data, par- 
a
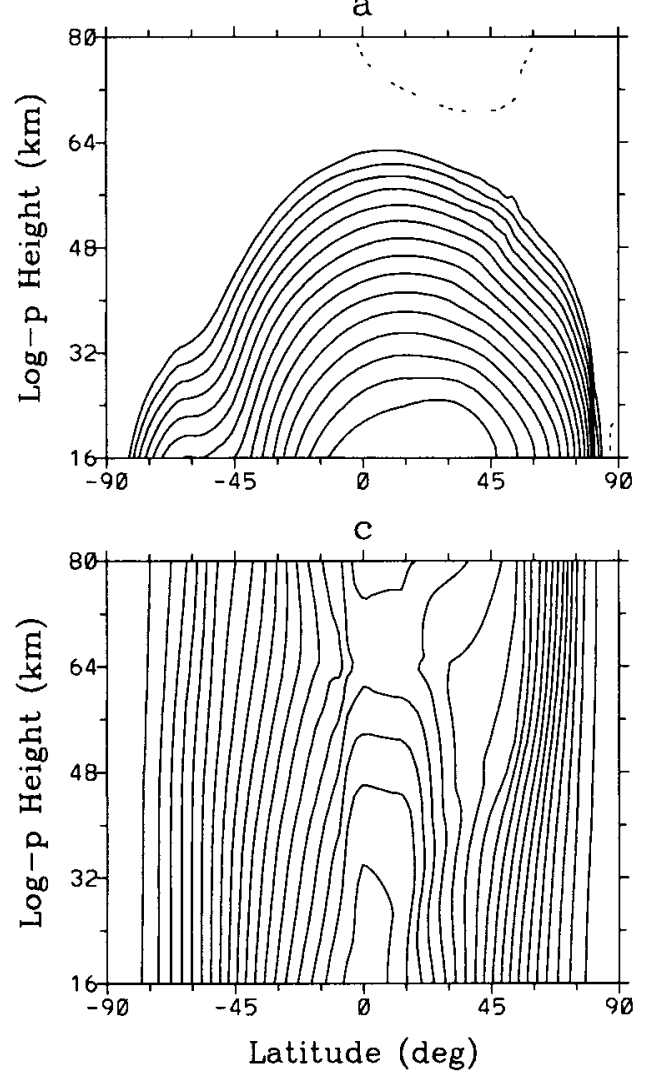

$\mathrm{b}$
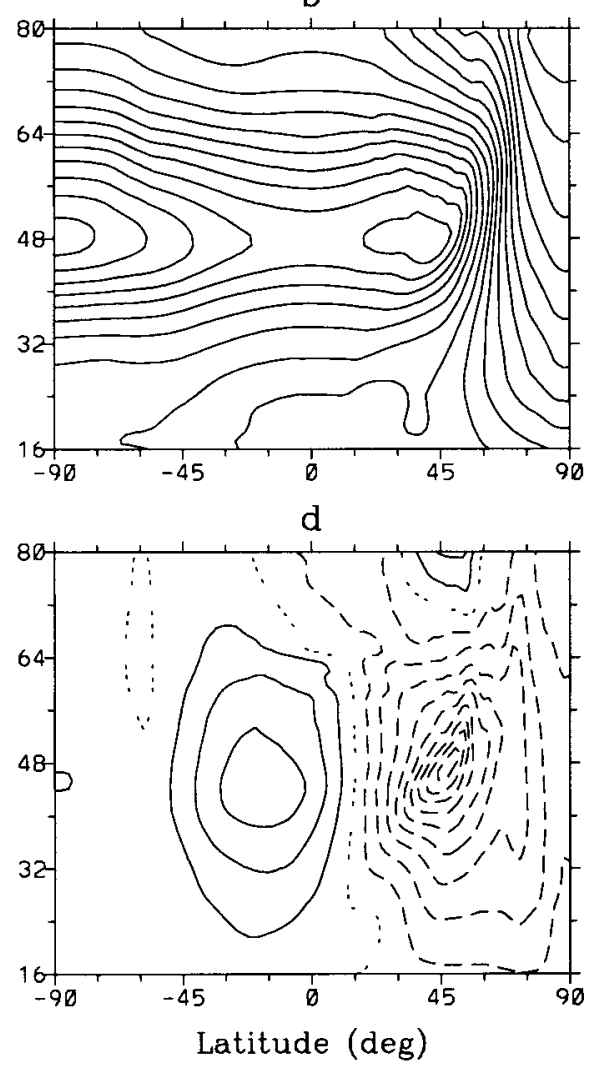

FIG. 12. Same as Fig. 11 but with $T_{\text {rad }}$ based on CIRA temperatures. Contour interval in (b) is $8 \mathrm{~K}$.

ticularly tropical winds, these comprehensive models provide a proxy dataset. At the same time, such analysis is a useful test of the physical realism of a GCM.

The accuracy of zonal-mean tropical gradient-wind balance was considered using a mechanistic model in section 3. Here we consider the Canadian Middle Atmosphere Model (CMAM) (documented in Beagley et al. 1997) in a similar light. The monthly averaged root- mean square of the difference between the two dominant terms in the zonal-mean meridional momentum equation, namely, the meridional gradient of the geopotential and the Coriolis torque term, is shown in Fig. 15 for the January zonal-mean state. It appears that gradientwind balance is a good approximation over most of the stratosphere and begins to get weaker in the upper stratosphere and mesosphere where the cross-equatorial flow
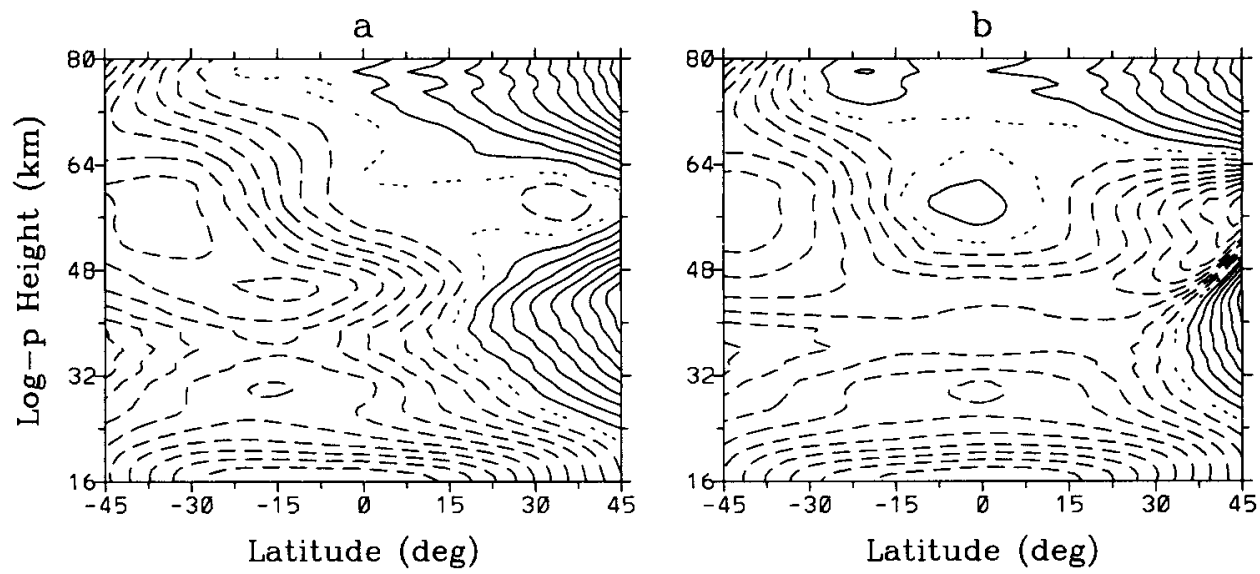

FIG. 13. Contour plot of (a) $\bar{T}_{\text {CIRA }}-T_{\text {rad }}$ and (b) $\bar{T}_{\text {CIRA }}-\bar{T}_{\text {mod }}$, with $\bar{T}_{\text {mod }}$ as in Fig. 8b. Contour interval is $2.5 \mathrm{~K}$ in both panels. 
a

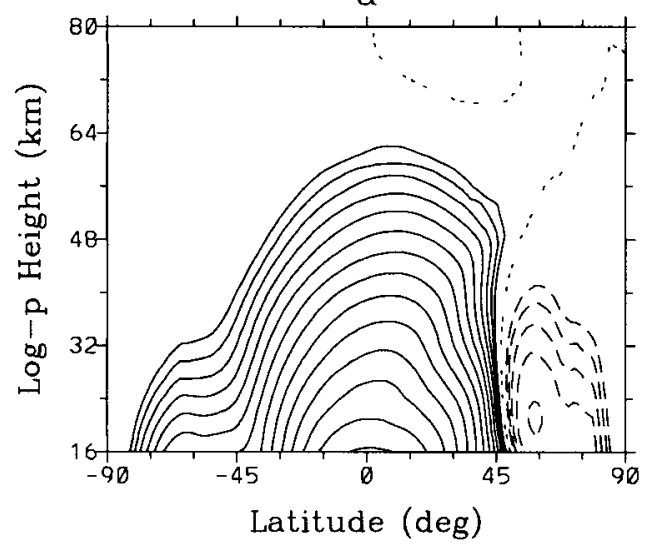

$\mathrm{b}$

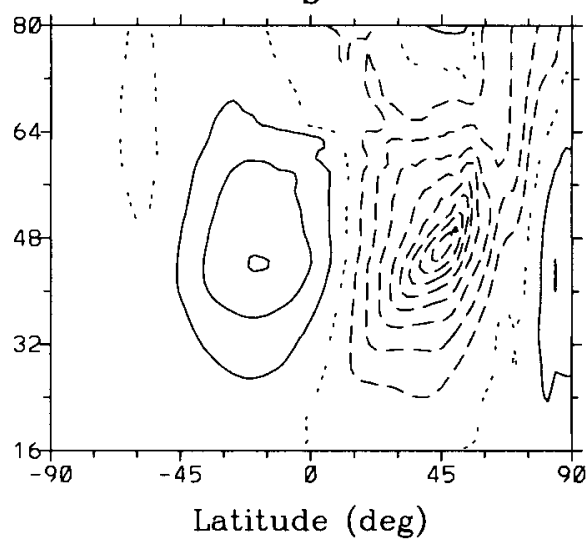

FIG. 14. Contour plot of (a) $\psi$ and (b) Q corresponding to the difference between Figs. 11 and 12 for the respective fields. To be compared with (a) and (d) of Fig. 2 (same contour interval).

is the largest. Nevertheless, the breakdown of balance is relatively slight in the monthly mean.

Inertial instability is active in the upper stratosphere of CMAM as indicated by the presence of $2 \Delta z$ cells (2-grid structures) (Fig. 16). These cells have a strong zonally symmetric component mainly on the winter side of the equator, similar to those found by Hunt (1981). The fact that the cells are on the grid scale in the vertical is not a reflection of computational instability; rather, it reflects the fact that the physical instability has no vertical scale selection in the absence of diffusion. Since explicit vertical diffusion is small in CMAM the instability occurs on the smallest allowable vertical scale (i.e., it is limited by grid diffusion). The latitudinal zonal wind profile at altitudes in the middle and upper stratosphere indicates the presence of inertial adjustment in CMAM. In particular, a kink in the zonal wind forms on the winter side of the equator (Fig. 17) that allows the zonal wind to satisfy the inertial stability criterion and that cannot be explained by radiative forcing or wave drag.

However, there are no parabolic regions in the stratosphere and above near the equator. Unlike the balance model parameterization, the explicitly resolved inertial adjustment in CMAM does not homogenize $\bar{m}$ in regions of inertial instability and only weakens $\partial \bar{m} / \partial \phi$ in small bands on both sides of the equator. There is certainly no indication of $\bar{m}$ homogenization occurring at higher latitudes such as in the balance model simulations of section 3. The absence of $\bar{m}$ homogenization suggests that there is significant damping by wave drag in the tropical upper stratosphere and mesosphere. The presence of a strong SAO in CMAM (Beagley et al. 1997) is consistent with this hypothesis.

The CMAM temperature field likewise gives an indication of the presence of the Hadley circulation. The
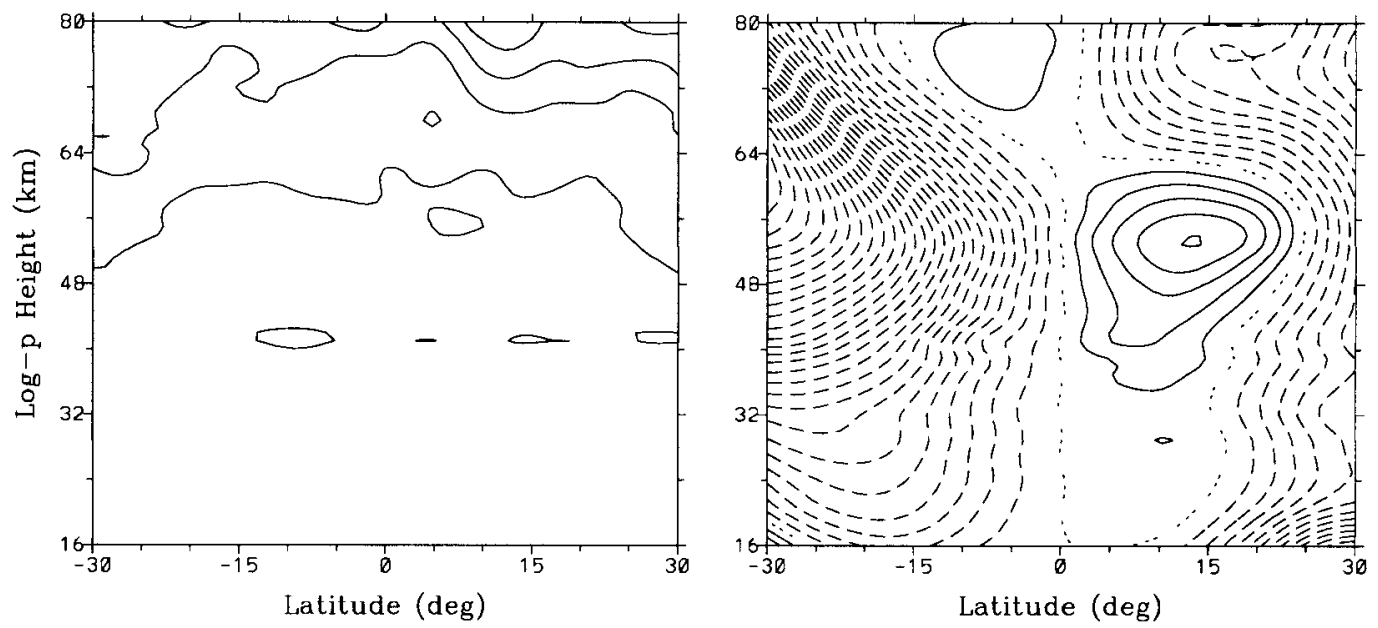

FIG. 15. Latitude-height plot of the Jan average of the rms gradient-wind imbalance of zonal mean fields from CMAM (left panel), and Jan mean $a^{-1} \partial \Phi / \partial \phi\left(\Phi\right.$ is the geopotential) (right panel); contour interval is $2 \times 10^{-4} \mathrm{~m}$ $\mathrm{s}^{-2}$ in both panels. There is little breakdown of gradient wind balance in the CMAM tropical stratosphere and lower mesosphere. 


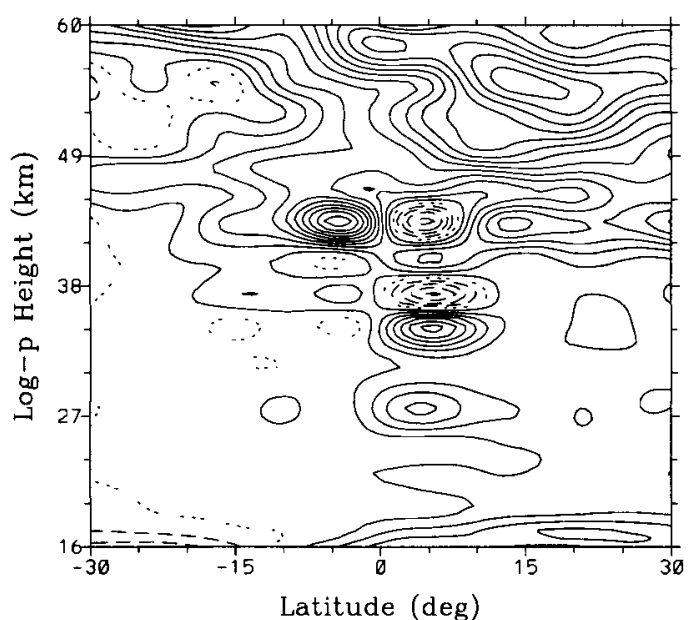

FIG. 16. CMAM Jan mean zonal-mean meridional residual velocity field; the contour interval is $0.2 \mathrm{~m} \mathrm{~s}^{-1}$. The presence of inertial instability is indicated by grid-trapped circulation cells near the equator (stacked layers of alternating poleward and equatorward flow). In the course of the annual cycle these cells alternate from one side of the equator to the other, minimizing during equinoxes.

latitudinal profile of the diabatic heating (Fig. 18, solid curve) reveals that the tropical heating maximum is in the summer hemisphere, as it is in the diabatic heating inferred from observed temperature (Fig. 18, dashed curve). Such a temperature profile can be explained by the superposition of the heating dipole of the Hadley circulation and the tropical heating distribution produced by wave drag. Summer hemisphere dynamical heating is too weak (Rosenlof 1995) to pull the temperature away from radiative equilibrium to this extent, while winter hemisphere drag would produce a heating maximum on the winter side of the equator (or on the equator if it is sufficiently close to it). In the lower stratosphere, the circulation and its associated diabatic heating are weak and therefore unable to produce a large redistribution of the upwelling and heating. At these

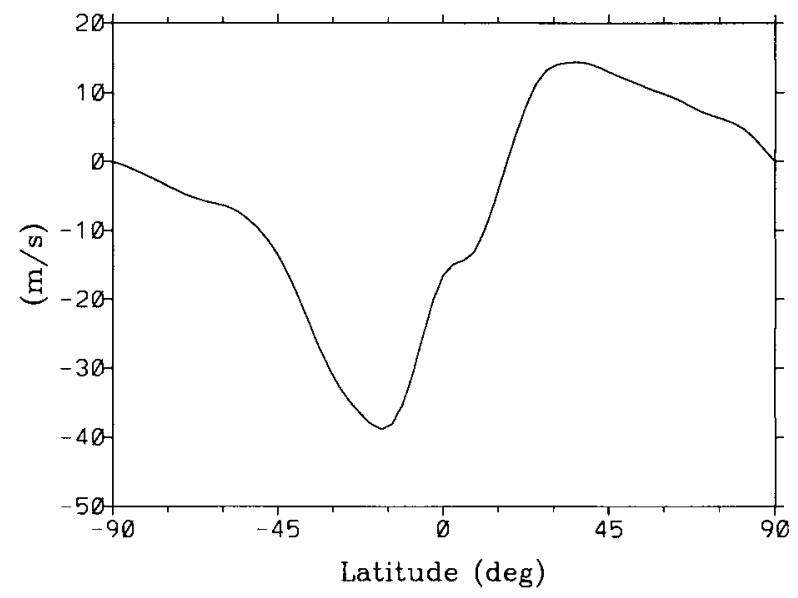

FIG. 17. Latitudinal profile of CMAM Jan mean $\bar{u}$ at $37 \mathrm{~km}$.

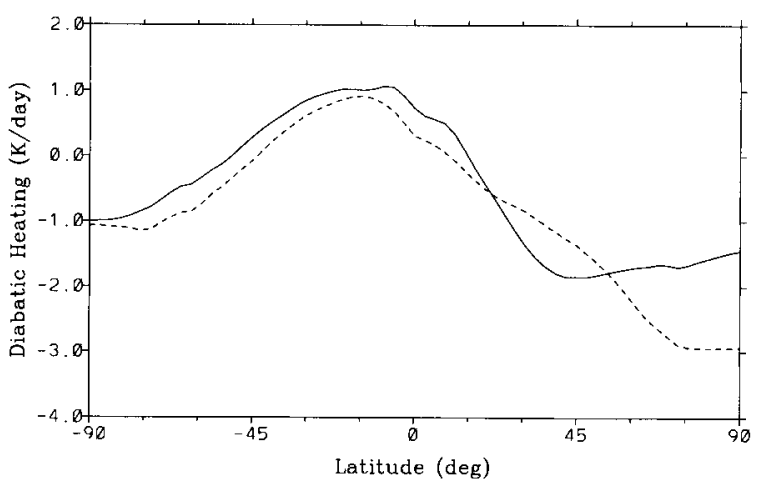

FIG. 18. Latitudinal profile of CMAM Jan mean diabatic heating at $37 \mathrm{~km}$ (solid) and observed Jan mean diabatic heating (based on CIRA $\bar{T}$ and the comprehensive radiative transfer scheme described in the text) at $37 \mathrm{~km}$ (dash).

levels the equatorial asymmetry of the upwelling is much smaller (cf. Figs. 9 and 10 of Randel et al. 1998).

A major feature of the tropical $\bar{m}$ distribution in both CMAM simulations and COSPAR International Reference Atmosphere (CIRA) (Flemming et al. 1990) observations is the region of overturned contours in the stratopause region (Figs. 6 and 19). This is another possible indication of the presence of the Hadley circulation in CMAM. As shown in section 3, horizontal deformation of $\bar{m}$ contours in the tropical stratopause region can be produced by the Hadley circulation. However, the degree of $\bar{m}$ contour deformation in both observations and CMAM suggests that this feature is the combined result of the Hadley circulation and the WDC (cf. Fig. 12). The necessary WDC can be plausibly produced by the climatologically inferred wave drag in the upper stratosphere, which has close proximity to the equator and is of large amplitude (Rosenlof 1995).

\section{Conclusions}

The results of this study support the work of Dunkerton (1989) in establishing the Hadley circulation as a significant part of the dynamics of the tropical middle atmosphere during solstice seasons. It appears to be as-

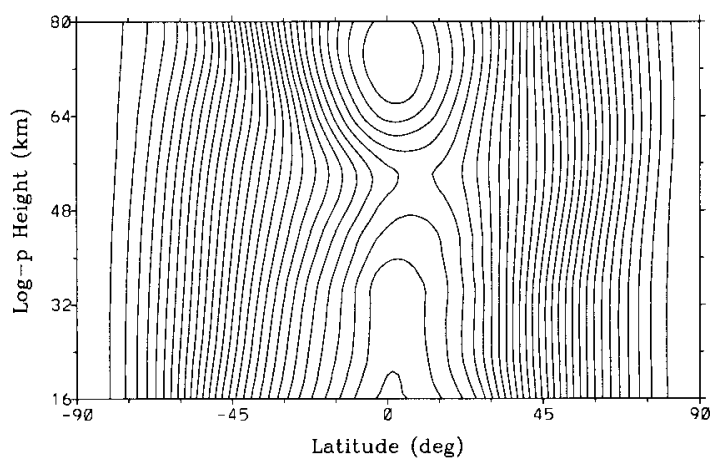

FIG. 19. Jan mean CMAM absolute angular momentum distribution $\left(10^{8} \mathrm{~m}^{2} \mathrm{~s}^{-1}\right)$. 
sociated with at least half of the diabatic heating (Semeniuk and Shepherd 2001; Fig. 21) and nearly all of its equatorial asymmetry (Fig. 13) in the tropical upper stratosphere. This underscores the significance of the Hadley circulation for the structure of the tropical state. The total diabatic circulation pattern is heavily modified thereby, with the upwelling maximum shifting into the summer hemisphere. The contribution of the Hadley circulation in the tropical lower stratosphere and mesosphere is, however, obscured by the effects of tropical wave drag and the wave-driven circulation.

If the gradient-wind balance state is only partly achieved in reality, one would expect the Hadley circulation to be weaker than predicted by the balance model. However, based on simulations using a mechanistic primitive equations model, balance appears to be sufficiently robust. Even though there is formation of intense inertial-adjustment-related circulation cells, they can be regarded as superimposed on a balanced basic state (as indicated by the simple difference of the left and right panels of Fig. 9). In CMAM, gradientwind balance starts to break down gradually in the stratopause region and above (Fig. 15), which is consistent with the decrease in dynamical timescales with altitude.

Dunkerton (1991) argued that the pattern of severe $\bar{m}$ isopleth deformation near the stratopause was partly the result of extratropical wave drag. It is clear that this deformation, which is most prominant during solstices, is due to significant cross-equatorial flow into the winter hemisphere. However, the location of this flow is partly the result of the concentration near the stratopause of the diabatic heating that produces the Hadley circulation. The advection of $\bar{m}$ by the Hadley circulation becomes significant at these altitudes. In contrast, the meridional velocity in the middle and lower stratosphere is too weak to produce significant overturning of $\bar{m}$ isopleths. As a result, the meridional streamfunction equation is nearly parabolic in the tropical stratopause region, which leads to the enhancement of the wavedriven circulation. This effect is considered in more detail in Semeniuk and Shepherd (2001).

The observed flattening of $\bar{m}$ isopleths in the tropical stratopause region implies that cross-equatorial flow must dominate local stabilization by tropical wave drag. The reason why severe $\bar{m}$ isopleth deformation does not extend through the mesosphere in reality (e.g., Fig. 6) is likely due to the increasing amplitude of tropical gravity wave drag with altitude. Similar stabilization by wave drag should occur in the stratosphere as well, although to a lesser extent.

Extratropical wave drag affects the Tropics through the wave-driven circulation in an opposing manner to that of tropical wave drag, if the latter can be regarded as acting to damp the zonal wind. Streamlines of the diabatic circulation have a significant horizontal component in the Tropics in the course of the annual cycle. So qualitatively, the wave-driven circulation adds to the cross-equatorial flow produced by the Hadley circula- tion in the upper stratosphere. This translates into more overturning of tropical $\bar{m}$ isopleths, as indicated by the results of section 4 . There is reason to believe that planetary wave drag reaches deep into the Tropics in the upper stratosphere and mesosphere, which increases the impact of the wave-driven circulation on the Tropics. On the other hand, at higher latitudes there is significant stabilization of $\bar{m}$ isopleths by planetary wave drag. So the significant $\bar{m}$ homogenization seen in the unforced balance model in the subtropics and higher latitudes does not occur in nature.

The direct impact of the wave-driven circulation on the solstitial Hadley circulation is limited by the nature of the balance system, which prevents the former from producing a meridional temperature gradient near the equator. As a result, the wave-driven circulation cannot affect the temperature near the equator in a way that counteracts or enhances the formation of the diabatic heating that drives the Hadley circulation. In the linearized system the two types of circulation exist independently of each other (assuming that the diabatic heating is a linear function of temperature). So any interaction that occurs is purely nonlinear and primarily involves the advection of $\bar{m}$. Except for the tropical stratopause region, nonlinearity is relatively weak and the $\bar{m}$ distribution is close to the linear case.

The gradient-wind balance constraint on the meridional temperature profile near the equator is not sufficient to determine the intensity and distribution of the Hadley circulation. The circulation that forms as a result of the inability of the system to relax to radiative equilibrium in the Tropics advects the zonal wind toward the winter pole. This results in both a shift in the diabatic heating toward the winter hemisphere and its intensification, which in turn amplifies the Hadley circulation. This selfgeneration by the Hadley circulation eventually saturates, but the ultimate state can be significantly affected by the presence of other processes such as inertial adjustment or wave drag.

A potentially critical limitation of the balance model used here is the inertial adjustment scheme. Its appropriateness has been tested in section 3 by direct comparison with a primitive equations model, with close agreement. Compared to the CIRA and CMAM $\bar{m}$ distributions, on the other hand, the angular-momentumconserving balance model adjustment scheme appears to be too efficient. Part of this may be the result of the absence of important tropical wave drag in the balance model. However, it also could be related to the physics of inertial adjustment, which is not instantaneous in reality. Further investigation of the phenomenology of inertial adjustment would be welcome.

It should also be noted that ultimately the radiative equilibrium temperature is partly the result of wavedriven transport of ozone and other trace gases. Neglect of this feedback is an implicit approximation of the modeling conducted here. However, it is unlikely that solstitial meridional solar heating gradients in the Trop- 
ics are dependent on transport by middle-atmosphere wave drag.

Acknowledgments. We thank T. J. Dunkerton for his extensive comments on the original manuscript, and $\mathrm{R}$. A. Plumb for useful feedback. This work represents part of KS's Ph.D. thesis at the University of Toronto. He wishes to acknowledge support from an Ontario Graduate Scholarship, the Canadian MAM project, and the university. TGS is supported by the Natural Sciences and Engineering Research Council and the Meteorological Service of Canada.

\section{APPENDIX A}

\section{The Streamfunction Equation}

To be able to use both prognostic equations in the numerical model requires a discretization scheme that preserves thermal-wind balance. The scheme used here is motivated by that of Kinnersley (1996). The numerical model streamfunction is obtained from the discretized governing equations using the thermal-wind balance condition discretized at the end of the time step. Discretizing the continuous streamfunction amounts to using the thermal-wind balance relation discretized at the beginning of the time step. This method gives a nonlinear streamfunction when using $\bar{u}$ as one of the prognostic variables since the thermal-wind relation is nonlinear in $\bar{u}$. The nonlinearity can be avoided by using a variable that makes the thermal-wind relation linear. In particular, the zonal wind is replaced by $M=(\bar{m} /$ $a)^{2}$, where $\bar{m}=a \cos \phi(\bar{u}+a \Omega \cos \phi)$. Under climatological conditions $\bar{m}>0$ so $\bar{u}$ can be easily obtained from $M$. With the zonal wind equation replaced by an equation for $M$, the streamfunction equation coefficients are

$$
\begin{aligned}
A & =\frac{R \cos ^{2} \phi}{H a^{2}} S \\
B & =\frac{2 \tan \phi}{a} \frac{\partial M}{\partial z} \\
C & =-\frac{\tan \phi}{a^{2}} \frac{\partial M}{\partial \phi} \\
D & =\left[(1-\kappa) \frac{\partial M}{\partial z}+R \cos ^{2} \phi S\right] \frac{\tan \phi}{H a^{2}} \\
E & =\left(\frac{1}{a^{2} \cos { }^{2} \phi}+\frac{3 \tan ^{2} \phi}{a^{2}}\right) \frac{\partial M}{\partial z}-\frac{\tan \phi}{H a^{2}} \frac{\partial M}{\partial \phi} \\
F & =\rho_{0} \cos \phi\left[\frac{2 \sin \phi}{a} \frac{\partial(\sqrt{M} \mathcal{F})}{\partial z}+\frac{R \cos ^{2} \phi}{H a} \frac{\partial Q}{\partial \phi}\right]
\end{aligned}
$$

where $S=\partial \bar{T} / \partial z+(\kappa / H) \bar{T}$. The discretized version of $F$ has an additional correction term, which originates from the thermal-wind-balance-conserving discretization scheme, namely,

$$
F \rightarrow F+\frac{\rho_{0} \cos \phi}{\Delta t}\left(\frac{\tan \phi}{a} \frac{\partial M}{\partial z}+\frac{R \cos ^{2} \phi}{H a} \frac{\partial \bar{T}}{\partial \phi}\right),
$$

where $\Delta t$ is the time step. The term in the brackets is the deviation from thermal-wind balance, and is equal to zero in the continuous system.

\section{APPENDIX B}

\section{Inertial Adjustment Scheme}

The inertial adjustment scheme is an iterative redistribution of $\bar{u}$ at every time step at levels where the condition $f(\partial \bar{u} / \partial \phi-a f)>0$ is satisfied. The version used here is a simplified form of the one used by Holton and Wehrbein (1980). In the original scheme, $\bar{u}_{\phi \phi}$ is modified where the zonal wind satisfies the barotropic instability criterion. However, barotropic instability is a separate issue and is not an essential element of tropical dynamics, so we ignore this possibility.

It was found that using $f+\bar{u} \tan \phi / a$ instead of $f$ in the determination of the region of symmetric instability led to some numerical instability. Since there was not much difference in the regions requiring adjustment either way, the simpler form was used.

The discrete system domain is rectangular and defined by $\left\{(i, j) \mid 1 \leq i \leq N_{\phi}, 1 \leq j \leq N_{z}\right\}$. Here, $i$ is the latitudinal index and $j$ is the vertical index. The model grid was 65 by 65 . For every $j$ on the domain grid, ranges of $i$ where the inertial stability condition is violated are identified at every time step and take the form $\left\{N_{\phi 1}(j) \leq i \leq N_{\phi 2}(j)\right\}$. On $\left\{N_{\phi 1}, N_{\phi 2}\right\}$ the discretized zonal-wind field $\bar{u}_{i j}$ undergoes a conservative iterative adjustment of the form

$$
\begin{gathered}
\bar{u}_{i j} \rightarrow \bar{u}_{i j}+\frac{1}{2}\left(\bar{u}_{i+1 j}-\bar{u}_{i j}-a \Delta \phi f\right) \\
\bar{u}_{i+1 j} \rightarrow \bar{u}_{i+1 j}-\frac{1}{2}\left(\bar{u}_{i+1 j}-\bar{u}_{i j}-a \Delta \phi f\right)
\end{gathered}
$$

in the NH until $\partial \bar{u} / \partial \phi=a f$ is satisfied $(\Delta \phi$ is the latitudinal grid spacing). The iteration consists of repeated loops over index $i$ in ascending order; no vertical adjustment is performed. In the $\mathrm{SH}$ the scheme involves the iteration looping over index $i$ in descending order and the substitution $i+1 \rightarrow i-1$ and $i \rightarrow i$ in the expressions above (for the terms in brackets $i+1 \rightarrow$ $i$ and $i \rightarrow i-1)$. This scheme leads to the accumulation of angular momentum near the equator with a very sharp latitudinal gradient at $\phi=0$. This gradient is removed by an ad hoc extension of the adjustment region into the opposite hemisphere where $f$ is replaced by a function rapidly decaying to zero.

The discretized temperature field $\bar{T}_{i j}$ is also iteratively adjusted at every time step to be in thermal-wind balance with the redistributed $\bar{u}_{i j}$. 


\section{REFERENCES}

Andrews, D. G., J. R. Holton, and C. B. Leovy, 1987: Middle Atmosphere Dynamics. Academic Press, 489 pp.

Beagley, S. R., J. de Grandpré, J. N. Koshyk, N. A. McFarlane, and T. G. Shepherd, 1997: Radiative-dynamical climatology of the first-generation Canadian middle atmosphere model. Atmos.Ocean, 35, 293-331.

Clark, P. D., and P. H. Haynes, 1996: Inertial instability on an asymmetric low-latitude flow. Quart. J. Roy. Meteor. Soc., 122, 151182.

Dunkerton, T. J., 1983: A nonsymmetric equatorial inertial instability. J. Atmos. Sci., 40, 807-813.

_ 1989: Nonlinear Hadley circulation driven by asymmetric differential heating. J. Atmos. Sci., 46, 956-974.

_ 1991: Nonlinear propagation of zonal winds in an atmosphere with Newtonian cooling and equatorial wavedriving. J. Atmos. Sci., 48, 236-263.

Eliassen, A., 1951: Slow thermally or frictionally controlled meridional circulation in a circular vortex. Astrophys. Norv., 5, 1960.

Fels, S. B., 1987: Response of the middle atmosphere to changing $\mathrm{O}_{3}$ and $\mathrm{CO}_{2}-\mathrm{A}$ speculative tutorial. Transport Processes in the Middle Atmosphere, G. Visconti and R. Garcia, Eds., D. Reidel Publishing, 371-386.

Flemming, E. L., S. Chandra, J. J. Barnett, and M. Corney, 1990: Zonal mean temperature, pressure, zonal wind, and geopotential height as functions of latitude. Adv. Space Res., 10 (12), 11-59.

Fomichev, V. I., and J.-P. Blanchet, 1995: Development of the new CCC/GCM longwave radiation model for extension into the middle atmosphere. Atmos.-Ocean, 33, 513-529.

Garcia, R. R., 1991: Parameterization of planetary wave breaking in the middle atmosphere. J. Atmos. Sci., 48, 1405-1419.

Haynes, P. H., C. J. Marks, M. E. McIntyre, T. G. Shepherd, and K. P. Shine, 1991: On the "downward control" of extratropical diabatic circulations by eddy-induced mean zonal forces. J. Atmos. Sci., 48, 651-678.

Held, I. M., and A. Y. Hou, 1980: Nonlinear axially symmetric circulations in a nearly inviscid atmosphere. J. Atmos. Sci., 37, $515-533$.
Holton, J. R., and W. M. Wehrbein, 1980: A numerical model of the zonal mean circulation of the middle atmosphere. Pure Appl. Geophys., 118, 284-306.

- P. H. Haynes, M. E. McIntyre, A. R. Douglass, R. B. Rood, and L. Pfister, 1995: Stratosphere-troposphere exchange. Rev. Geophys., 33, 403-439.

Hunt, B. G., 1981: The maintenance of the zonal mean state of the upper atmosphere as represented in a three-dimensional general circulation model extending to $100 \mathrm{~km}$. J. Atmos. Sci., 38, 21722186.

Kinnersley, J. S., 1996: The climatology of the stratospheric 'THIN AIR' model. Quart. J. Roy. Meteor. Soc., 122, 219-252.

Plumb, R. A., and J. Eluszkiewicz, 1999: The Brewer-Dobson circulation: Dynamics of the tropical upwelling. J. Atmos. Sci., 56, 868-890.

Randel, W. J., F. Wu, J. M. Russell III, A. Roche, and J. W. Waters, 1998: Seasonal cycles and QBO variations in stratospheric $\mathrm{CH}_{4}$ and $\mathrm{H}_{2} \mathrm{O}$ observed in UARS HALOE data. J. Atmos. Sci., 55, 163-185.

Rosenlof, K. H., 1995: Seasonal cycle of the residual mean meridional circulation in the stratosphere. J. Geophys. Res., 100, 51735191

Saravanan, R., 1992: A mechanistic spectral primitive equation model using pressure coordinates. [Software and documentation available from R. Saravanan, NCAR, P.O. Box 3000, Boulder, CO 80307.]

Scott, R. K., and P. H. Haynes, 1998: Internal interannual variability of the extratropical stratospheric circulation: The low-latitude flywheel. Quart. J. Roy. Meteor. Soc., 124, 2149-2173.

Semeniuk, K., and T. G. Shepherd, 2001: Mechanisms for tropical upwelling in the stratosphere. J. Atmos. Sci., 58, 3097-3115.

Shepherd, T. G., K. Semeniuk, and J. N. Koshyk, 1996: Sponge layer feedbacks in middle-atmosphere models. J. Geophys. Res., 101, 23 447-23 464.

Stevens, D. E., 1983: On the symmetric stability and instability of zonal mean flows near the equator. J. Atmos. Sci., 40, 882-893.

Tung, K.-K., and J. S. Kinnersley, 2001: Mechanisms by which extratropical wave forcing in the winter stratosphere induces upwelling in the summer hemisphere. J. Geophys. Res., in press. 